\title{
Robust nonlinear control strategy to maximize energy capture in a variable speed wind turbine with an internal induction generator
}

\author{
Erhun IYASERE ${ }^{1}$, Mohammed H. SALAH ${ }^{2}$, Darren M. DAWSON ${ }^{3}$, John R. WAGNER ${ }^{4}$, Enver TATLICIOGLU ${ }^{5}$ \\ 1.Jacobsen, a Textron Company, Charlotte, NC, U.S.A.; \\ 2.Department of Mechatronics Engineering, Hashemite University, Zarqa, Jordan; \\ 3.Department of Electrical \& Computer Engineering, Clemson University, Clemson, SC, U.S.A.; \\ 4.Department of Mechanical Engineering, Clemson University, Clemson, SC, U.S.A.; \\ 5.Department of Electrical \& Electronics Engineering, Izmir Institute of Technology, Izmir, Turkey
}

\begin{abstract}
This paper proposes a control strategy to maximize the wind energy captured in a variable speed wind turbine, with an internal induction generator, at low to medium wind speeds. The proposed strategy controls the tip-speed ratio, via the rotor angular speed, to an optimum point at which the efficiency constant (or power coefficient) is maximum for a particular blade pitch angle and wind speed. This control method allows for aerodynamic rotor power maximization without exact wind turbine model knowledge. Representative numerical results demonstrate that the wind turbine can be controlled to achieve near maximum energy capture.
\end{abstract}

Keywords: Robust nonlinear control; Maximum energy capture; Wind turbine

\section{Introduction}

Wind energy has evolved into an attractive energy source for electric utilities, even though it is currently responsible for about only one percent of the global electrical power production. The structure of wind turbines, as well as the fact that the wind energy rate is uncontrollable, complicates the problem of regulating power capturing. This engineering challenge has been alleviated by the construction of variable speed wind turbines, which are designed to regulate the power captured over a range of operating speeds. However, the efficiency of power regulation is dependent on the selected control method.

The standard region 2 (power capture maximization mode) control scheme used for variable speed wind turbines ( $\tau=k \omega^{2}$, where $\tau$ is the control torque, $\omega$ is the rotor angular speed and $k$ is a control gain) has some disadvantages that can result in unsatisfactory power capture. First, the control gain, $k$, is difficult to be determined due to the dependence on exact model knowledge (maximum power efficiency constant and optimal tip-speed ratio). Second, the standard value of $k$ might not provide the maximum energy capture under real world turbulent conditions. Johnson and Fingersh [1] showed via numerical simulation that smaller values of $k$ than the standard can result in increased power capture. They proposed a new control scheme, specifically, an adaptive control scheme that allowed for maximum power capture in the presence of parametric uncertainty. Similar adaptive control techniques for wind turbine control were developed in [2] and [3].

Other wind turbine control methods such as classical con- trol techniques [4-7], nonlinear control [8-9], robust control [10], fuzzy logic control [11-12], and intelligent control [13-14] have been utilized to regulate rotor speed and pitch angle and to enhance energy capture. Iyasere et al. [10] proposed a robust control strategy to control the blade pitch angle and rotor speed in a variable speed variable pitch wind turbine in order to maximize the energy capture, without the knowledge of the optimal tip-speed ratio and in the presence of model structural uncertainties.

An area of particular importance is the control of the internal generators used in wind turbines [15]. The most commonly used generator is the induction generator, of which the types include cage, wound rotor and doubly fed induction generator (DFIG). The dynamic modeling [16-20] and control [21-28] of induction machines have been extensively researched. Thringer and Luomi [16] examined the validity of various dynamic models of induction machines to include the fifth-order Park model and other reduced order models by predicting the low frequency dynamic response of a $15 \mathrm{~kW}$ induction machine and comparing results to actual measurements. They concluded that the Park model accurately predicts rotor speed, electrical torque, active power, reactive power and stator current responses to perturbations in the shaft torque, supply frequency and voltage magnitude. In power system analysis, a third-order model was determined to be the right fit for accuracy and simplicity. Tapia et al. [17] developed the mathematical model of a grid connected wind driven DFIG and presented a comparison of the simulation results to real machine performance results. They also developed a stator-

Received 23 December 2010; revised 3 May 2011.

(c) South China University of Technology and Academy of Mathematics and Systems Science, CAS and Springer-Verlag Berlin Heidelberg 2012 
flux-oriented vector control based technique to control the generator power factor. Mullane and O'Malley [18] examined the inertial response of a squirrel cage and a doubly fed induction wind turbine generator using fifth-order induction generator models. They discovered that a DFIG utilizing field-oriented control is strongly influenced by rotor current controller bandwidth. Hu and Dawson [21] presented an adaptive partial state feedback position tracking controller for the full-order nonlinear dynamic model for an induction motor. The controller compensates for uncertainty in rotor resistance and mechanical system parameters while yielding asymptotic rotor position tracking. Datta and Ranganathan [22] developed a simple position-sensorless strategy for rotor-side field-oriented control of a wound rotor induction machine. The algorithm is based on axis transformation with reduced dependence on machine parameters compared to other methods. Pena et al. [23] described a vector control scheme for the supply-side voltage sourceconverter of a DFIG for independent control of active and reactive power. This strategy was embedded into an optimal tracking controller in order to maximize energy capture in a wind energy application. Two tracking schemes were developed: speed mode and current mode.

In this study, a control strategy is developed to regulate the rotor speed of a small variable speed wind turbine system with an induction generator. The control objective is to maximize the energy captured by the wind turbine for low to medium air speeds by tracking a desired rotor speed in the presence of system nonlinearities and structural uncertainty. Additionally, the maximization of the energy captured is achieved without the knowledge of the relationship that governs the power capture efficiency of the wind turbine. Instead, an optimization algorithm is developed to seek the unknown optimal rotor speed that maximizes the energy captured (via the aerodynamic rotor power), at a particular blade pitch angle and wind speed. The problem of not explicitly knowing the rotor speed a priori is countered by the fact that the optimal rotor speed changes as the wind speed changes which may be accommodated for by choosing the right optimization algorithm. A robust controller is designed and proven to yield a globally uniformly ultimately bounded (GUUB) stable closed loop system through Lyapunov-based analysis.

The rest of the paper is organized as follows. In Section 2, the system model and problem statement are mathematically formulated. In Section 3, a robust nonlinear speed tracking controller is designed based on a Lyapunov stability analysis. In Section 4, an observer is designed to estimate the system nonlinearities. In Section 5, the estimate of the system nonlinearities is utilized to generate the rotor speed reference trajectory followed by numerical results in Section 6. Finally, concluding remarks are presented in Section 7.

\section{Wind turbine dynamic model}

A typical wind turbine model consists of a wind rotor, drive shaft and an internal induction generator. The aerody- namic power captured by the wind, $P_{\text {aero }}(t) \in \mathbb{R}^{+}$, can be defined as ${ }^{1}$

$$
P_{\text {aero }} \triangleq \frac{1}{2} C_{\mathrm{p}} \rho_{\mathrm{a}} A v_{\mathrm{a}}^{3},
$$

where $\rho_{\mathrm{a}} \in \mathbb{R}^{+}$is the air density and $A \in \mathbb{R}^{+}$is the rotor swept area, which is equal to $\pi R_{\mathrm{b}}^{2}$ where $R_{\mathrm{b}} \in \mathbb{R}^{+}$is the blade length. The variable $v_{\mathrm{a}}(t) \in \mathbb{R}^{+}$represents the wind speed, and $C_{\mathrm{p}}(\lambda, \beta) \in \mathbb{R}$ denotes the rotor power coefficient of the wind turbine and it is a function of the tip-speed ratio, $\lambda(t) \in \mathbb{R}^{+}$, and the blade pitch angle, $\beta(t) \in \mathbb{R}^{+}$. The tip-speed ratio, $\lambda(t)$, is defined as

$$
\lambda \triangleq \frac{\omega R_{\mathrm{b}}}{v_{\mathrm{a}}},
$$

where $\omega(t) \in \mathbb{R}^{+}$is the rotor speed of wind turbine. In a variable speed fixed pitch wind turbine system, there exists a constant optimal rotor speed, denoted by $\omega^{*} \in \mathbb{R}^{+}$(and hence an optimal tip-speed ratio, $\lambda^{*} \in \mathbb{R}^{+}$), for a given pitch angle, $\beta$, and a particular wind speed, $v_{\mathrm{a}}$, at which the power capture efficiency is maximum. Hence, rotor power coefficient, $C_{\mathrm{p}}(\cdot)$, is maximum and represented as $C_{\mathrm{p}}^{\max }$ where $C_{\mathrm{p}}^{\max } \triangleq C_{\mathrm{p}}\left(\lambda^{*}, \beta\right)$ and $\lambda^{*} \triangleq \frac{\omega^{*} R_{\mathrm{b}}}{v_{\mathrm{a}}}$. The aerodynamic power captured by the rotor, $P_{\text {aero }}(t)$, can also be expressed as

$$
P_{\text {aero }} \triangleq \tau_{\text {aero }} \omega,
$$

where $\tau_{\text {aero }}(t) \in \mathbb{R}^{+}$is the aerodynamic torque applied to the rotor by the wind. An expression for $\tau_{\text {aero }}(t)$ can be derived from (1)-(3) as

$$
\tau_{\text {aero }} \triangleq \frac{1}{2} \rho_{\mathrm{a}} A R_{\mathrm{b}} \frac{C_{\mathrm{p}}}{\lambda} v_{\mathrm{a}}^{2} .
$$

Remark 1 Since the rotor power coefficient, $C_{\mathrm{p}}(\cdot)$, is eventually unknown, hence the aerodynamic torque, $\tau_{\text {aero }}(t)$, and then the aerodynamic power, $P_{\text {aero }}(t)$, are unmeasurable.

\subsection{Mechanical subsystem dynamics}

The mechanical subsystem that describes the rotor dynamics of the variable speed wind turbine can be of the following form:

$$
J \dot{\omega}+f=\tau_{\mathrm{em}},
$$

where $J \in \mathbb{R}^{+}$is the rotor moment of inertia, $\dot{\omega}(t) \in \mathbb{R}$ is the rotor acceleration, $f\left(\omega, v_{\mathrm{a}}\right) \in \mathbb{R}$ represents the system unknown nonlinearities and is defined as $f \triangleq-\tau_{\text {aero }}$, and $\tau_{\mathrm{em}} \in \mathbb{R}^{+}$is the electromagnetic torque and is considered as the torque control input for the generator.

\subsection{Electrical subsystem dynamics}

An induction generator is made by three stator windings and three rotor windings. A two-phase equivalent machine representation was introduced in [29] and is utilized in this paper with the assumptions of equal mutual and autoinductances as well as a linear magnetic circuit. The electrical dynamics of the internal induction generator can be described by the following dynamic equations (for the exact transformation of three-phase variables into two-phase ones used in this paper, refer to [24]):

$$
\dot{\bar{\Psi}}_{\mathrm{s}}=-R_{\mathrm{s}} \bar{I}_{\mathrm{s}}+\bar{V}_{\mathrm{s}}
$$

\footnotetext{
1 Nomenclature and subscripts and superscripts can be found in Appendix A6.
} 


$$
\begin{aligned}
& \dot{\bar{\Psi}}_{\mathrm{r}}=-R_{\mathrm{r}} \bar{I}_{\mathrm{r}}+n_{\mathrm{p}} \omega \Delta \bar{\Psi}_{\mathrm{r}}+\bar{V}_{\mathrm{r}}, \\
& \bar{I}_{\mathrm{s}}=\kappa_{1} \bar{\Psi}_{\mathrm{s}}-\kappa_{2} \bar{\Psi}_{\mathrm{r}}, \\
& \bar{I}_{\mathrm{r}}=\kappa_{1} \bar{\Psi}_{\mathrm{r}}-\kappa_{2} \bar{\Psi}_{\mathrm{s}}, \\
& \tau_{\mathrm{em}}=\alpha \bar{\Psi}_{\mathrm{s}}^{\mathrm{T}} \Delta \bar{\Psi}_{\mathrm{r}}, \\
& \Delta \triangleq\left[\begin{array}{cc}
0 & -1 \\
1 & 0
\end{array}\right], \alpha \triangleq n_{\mathrm{p}} \kappa_{2},
\end{aligned}
$$

where $\bar{\Psi}_{\mathrm{s}} \triangleq\left[\begin{array}{ll}\bar{\Psi}_{\mathrm{S}_{\mathrm{a}}} & \bar{\Psi}_{\mathrm{s}_{\mathrm{b}}}\end{array}\right]^{\mathrm{T}}, \bar{\Psi}_{\mathrm{r}} \triangleq\left[\begin{array}{ll}\bar{\Psi}_{\mathrm{r}_{\mathrm{a}}} & \bar{\Psi}_{\mathrm{r}_{\mathrm{b}}}\end{array}\right]^{\mathrm{T}} \in \mathbb{R}^{2}$ are the stator and rotor flux linkage, respectively; $\bar{I}_{\mathrm{s}} \triangleq\left[\begin{array}{ll}\bar{I}_{\mathrm{a}} & \bar{I}_{\mathrm{sb}}\end{array}\right]^{\mathrm{T}}$, $\bar{I}_{\mathrm{r}} \triangleq\left[\begin{array}{ll}\bar{I}_{\mathrm{r}_{\mathrm{a}}} & \bar{I}_{\mathrm{r}_{\mathrm{b}}}\end{array}\right]^{\mathrm{T}} \in \mathbb{R}^{2}$ are the stator and rotor currents, respectively; and $\bar{V}_{\mathrm{s}} \triangleq\left[\begin{array}{ll}\bar{V}_{\mathrm{s}_{\mathrm{a}}} & \bar{V}_{\mathrm{S} \mathrm{b}}\end{array}\right]^{\mathrm{T}}, \bar{V}_{\mathrm{r}} \triangleq\left[\begin{array}{ll}\bar{V}_{\mathrm{r}_{\mathrm{a}}} & \bar{V}_{\mathrm{r}_{\mathrm{b}}}\end{array}\right]^{\mathrm{T}} \in \mathbb{R}^{2}$ are the stator and rotor voltages, respectively, where $\bar{V}_{\mathrm{s}}(t)$ is the voltage control input to be designed later. In (8) and (9), $\kappa_{1}, \kappa_{2} \in \mathbb{R}^{+}$are constants related to the generator parameters, and are given explicitly by

$$
\kappa_{1} \triangleq \frac{L_{\mathrm{s}}}{L_{\mathrm{s}}^{2}-M^{2}}, \quad \kappa_{2} \triangleq \frac{M}{L_{\mathrm{s}}^{2}-M^{2}} .
$$

To facilitate the control development, the following model characteristics are imposed.

Assumption 1 The system parameters $J, L_{\mathrm{s}}, M, n_{\mathrm{p}}$, $R_{\mathrm{b}}, R_{\mathrm{r}}, R_{\mathrm{s}}, \beta$ and $\rho_{\mathrm{a}}$ are assumed to be known constants.

Assumption 2 The variables $v_{\mathrm{a}}(t), \omega(t), \bar{I}_{\mathrm{s}}(t), \bar{I}_{\mathrm{r}}(t)$, and $\bar{V}_{\mathrm{s}}(t)$ are assumed to be measurable.

Assumption 3 The wind speed, $v_{\mathrm{a}}(t)$, is assumed to be constant or slowly time varying (i.e., $\dot{v}_{\mathrm{a}}(t) \cong 0$ ).

Assumption 4 The wind speed and its first two time derivatives, $v_{\mathrm{a}}(t), \dot{v}_{\mathrm{a}}(t)$, and $\ddot{v}_{\mathrm{a}}(t)$, are assumed to be bounded.

Assumption 5 As a consequence of the fact that $\tau_{\text {aero }}(t)$ is unmeasurable, the variable $f(\cdot)$, introduced in (5), is also unmeasurable.

Assumption 6 The variables, $f(\cdot), \dot{f}(\cdot)$, and $\ddot{f}(\cdot)$ are assumed to be bounded provided that their arguments are bounded.

Remark 2 The variable $f\left(\omega, v_{\mathrm{a}}\right)$ can be upper bounded by a known function such that $\left|f\left(\omega, v_{\mathrm{a}}\right)\right| \leqslant \rho_{\mathrm{z}}(\omega)$ where $\rho_{\mathrm{Z}}(\omega)$ is continuously differentiable for all $\omega(t)>0$.

\subsection{Electrical subsystem transformation}

An auxiliary control input $\omega_{\mathrm{s}}(t) \in \mathbb{R}$ is injected into the electrical subsystem dynamics via time-varying coordinate transformation [25] as follows:

$$
\left\{\begin{array}{l}
\Psi_{\mathrm{s}} \triangleq T \bar{\Psi}_{\mathrm{s}}, \quad I_{\mathrm{s}} \triangleq T \bar{I}_{\mathrm{s}}, \quad V_{\mathrm{r}} \triangleq T \bar{V}_{\mathrm{r}}, \\
\Psi_{\mathrm{r}} \triangleq T \bar{\Psi}_{\mathrm{r}}, \quad I_{\mathrm{r}} \triangleq T \bar{I}_{\mathrm{r}}, \quad V_{\mathrm{s}} \triangleq T \bar{V}_{\mathrm{s}},
\end{array}\right.
$$

where $T(t) \in \mathbb{R}^{2 \times 2}$ is defined as

$$
T \triangleq\left[\begin{array}{cc}
\cos \varepsilon_{0} & \sin \varepsilon_{0} \\
-\sin \varepsilon_{0} & \cos \varepsilon_{0}
\end{array}\right],
$$

where $\dot{\varepsilon}_{0}=\omega_{\mathrm{s}}$. Note that $T^{-\mathrm{T}} \Delta T^{-1}=\Delta$ and $\dot{T}=$ $-\Delta T \omega_{\mathrm{s}}$. The overall dynamics of the induction generator can then be given by the following model:

$$
\begin{aligned}
J \dot{\omega}+ & f=\tau_{\mathrm{em}}, \\
\dot{\Psi}_{\mathrm{s}}= & -R_{\mathrm{s}} \kappa_{1} \Psi_{\mathrm{s}}+R_{\mathrm{s}} \kappa_{2} \Psi_{\mathrm{r}}-\Delta \Psi_{\mathrm{s}} \omega_{\mathrm{s}}+V_{\mathrm{s}}, \\
\dot{\Psi}_{\mathrm{r}}= & -R_{\mathrm{r}} \kappa_{1} \Psi_{\mathrm{r}}+R_{\mathrm{r}} \kappa_{2} \Psi_{\mathrm{s}}+n_{\mathrm{p}} \omega \Delta \Psi_{\mathrm{r}}-\Delta \Psi_{\mathrm{r}} \omega_{\mathrm{s}} \\
& +V_{\mathrm{r}},
\end{aligned}
$$

$$
\begin{aligned}
& I_{\mathrm{s}}=\kappa_{1} \Psi_{\mathrm{s}}-\kappa_{2} \Psi_{\mathrm{r}}, \\
& I_{\mathrm{r}}=\kappa_{1} \Psi_{\mathrm{r}}-\kappa_{2} \Psi_{\mathrm{s}}, \\
& \tau_{\mathrm{em}}=\alpha \Psi_{\mathrm{s}}^{\mathrm{T}} \Delta \Psi_{\mathrm{r}},
\end{aligned}
$$

where

$$
\begin{aligned}
& \Psi_{\mathrm{s}} \triangleq\left[\begin{array}{ll}
\Psi_{\mathrm{s}_{1}} & \Psi_{\mathrm{s}_{2}}
\end{array}\right]^{\mathrm{T}} \in \mathbb{R}^{2}, \quad \Psi_{\mathrm{r}} \triangleq\left[\begin{array}{ll}
\Psi_{\mathrm{r}_{1}} & \Psi_{\mathrm{r}_{2}}
\end{array}\right]^{\mathrm{T}} \in \mathbb{R}^{2}, \\
& V_{\mathrm{r}} \triangleq\left[\begin{array}{ll}
V_{\mathrm{r}_{1}} & V_{\mathrm{r}_{2}}
\end{array}\right]^{\mathrm{T}} \in \mathbb{R}^{2}, \quad V_{\mathrm{s}} \triangleq\left[\begin{array}{ll}
V_{\mathrm{s}_{1}} & V_{\mathrm{s}_{2}}
\end{array}\right]^{\mathrm{T}} \in \mathbb{R}^{2} .
\end{aligned}
$$

\section{Nonlinear controller development}

\subsection{Control objectives}

The objective of the proposed controller is to maximize the aerodynamic rotor power of the wind turbine, $P_{\text {aero }}(t)$, by tracking a desired rotor speed, $\omega_{\mathrm{d}}(t) \in \mathbb{R}^{+}$, such that $\omega(t) \rightarrow \omega_{\mathrm{d}}(t)$ as $t \rightarrow \infty$. This is achieved in turn by tracking a desired electromagnetic torque, $\tau_{\mathrm{d}}(t) \in \mathbb{R}^{+}$, a desired stator flux $\Psi_{\mathrm{s}}^{\mathrm{d}}(t) \in \mathbb{R}^{2 \times 1}$, and a desired rotor flux $\Psi_{\mathrm{r}}^{\mathrm{d}}(t) \in \mathbb{R}^{2 \times 1}$ such that $\tau_{\mathrm{em}}(t) \rightarrow \tau_{\mathrm{d}}(t), \Psi_{\mathrm{s}}(t) \rightarrow \Psi_{\mathrm{s}}^{\mathrm{d}}(t)$, and $\Psi_{\mathrm{r}}(t) \rightarrow \Psi_{\mathrm{r}}^{\mathrm{d}}(t)$ as $t \rightarrow \infty$ where

$$
\begin{aligned}
& \Psi_{\mathrm{s}}^{\mathrm{d}} \triangleq\left[\begin{array}{ll}
\Psi_{\mathrm{s}_{1}}^{\mathrm{d}} & 0
\end{array}\right]^{\mathrm{T}}, \quad \Psi_{\mathrm{r}}^{\mathrm{d}} \triangleq\left[\begin{array}{ll}
\Psi_{\mathrm{r}_{1}}^{\mathrm{d}} & \Psi_{\mathrm{r}_{2}}^{\mathrm{d}}
\end{array}\right]^{\mathrm{T}}, \\
& \tau_{\mathrm{d}} \triangleq \alpha\left(\Psi_{\mathrm{s}}^{\mathrm{d}}\right)^{\mathrm{T}} \Delta \Psi_{\mathrm{r}}^{\mathrm{d}} .
\end{aligned}
$$

Remark 3 The desired rotor speed, $\omega_{\mathrm{d}}(t)$, is designedonline using a numerical-based optimization algorithm, as shown in Section 5, to maximize the rotor power, $P_{\text {aero }}(t)$, at a particular blade pitch angle, $\beta$, and wind velocity, $v_{\mathrm{a}}$, such that $\omega_{\mathrm{d}}(t) \rightarrow \omega^{*}$, where the optimal speed, $\omega^{*}$, is the result of the optimum seeking algorithm after convergence. Hence, $P_{\text {aero }}(t) \rightarrow P_{\max }$ if $\omega(t) \rightarrow \omega_{\mathrm{d}}(t)$. Additionally, $\omega_{\mathrm{d}}(t)$ is designed such that $\omega_{\mathrm{d}}(t), \dot{\omega}_{\mathrm{d}}(t)$, and $\ddot{\omega}_{\mathrm{d}}(t)$ are bounded.

\subsection{Error system development}

To quantify the control objectives, rotor speed tracking error, denoted by $e(t) \in \mathbb{R}$, as well as stator and rotor flux tracking errors, denoted by $\eta_{\mathrm{s}}(t), \eta_{\mathrm{r}}(t) \in \mathbb{R}^{2 \times 1}$, are defined as

$$
\begin{aligned}
& e \triangleq \omega_{\mathrm{d}}-\omega, \\
& \eta_{\mathrm{s}}=\left[\begin{array}{c}
\eta_{\mathrm{s}_{1}} \\
\eta_{\mathrm{s}_{2}}
\end{array}\right] \triangleq\left[\begin{array}{c}
\Psi_{\mathrm{s}_{1}}^{\mathrm{d}} \\
0
\end{array}\right]-\left[\begin{array}{l}
\Psi_{\mathrm{s}_{1}} \\
\Psi_{\mathrm{s}_{2}}
\end{array}\right], \\
& \eta_{\mathrm{r}}=\left[\begin{array}{l}
\eta_{\mathrm{r}_{1}} \\
\eta_{\mathrm{r}_{2}}
\end{array}\right] \triangleq\left[\begin{array}{c}
\Psi_{\mathrm{r}_{1}}^{\mathrm{d}} \\
\Psi_{\mathrm{r}_{2}}^{\mathrm{d}}
\end{array}\right]-\left[\begin{array}{l}
\Psi_{\mathrm{r}_{1}} \\
\Psi_{\mathrm{r}_{2}}
\end{array}\right],
\end{aligned}
$$

where $\eta_{\mathrm{s}_{1}}(t), \eta_{\mathrm{s}_{2}}(t), \eta_{\mathrm{r}_{1}}(t)$, and $\eta_{\mathrm{r}_{2}}(t) \in \mathbb{R}$. From the definition of the rotor speed tracking error in (23), and subsystem dynamics in (5), a rotor speed open-loop error dynamics is obtained as follows:

$$
J \dot{e}=J \dot{\omega}_{\mathrm{d}}+f-\tau_{\mathrm{em}} .
$$

By adding and subtracting $\tau_{\mathrm{d}}(t)$ to the right hand side of (26) and substituting (20) and (22), the expression of (26) becomes

$$
J \dot{e}=J \dot{\omega}_{\mathrm{d}}+f-\tau_{\mathrm{d}}+\alpha\left[\left(\Psi_{\mathrm{s}}^{\mathrm{d}}\right)^{\mathrm{T}} \Delta \Psi_{\mathrm{r}}^{\mathrm{d}}-\Psi_{\mathrm{s}}^{\mathrm{T}} \Delta \Psi_{\mathrm{r}}\right]
$$

The expression in (27) can be rewritten as

$$
\begin{gathered}
J \dot{e}=J \dot{\omega}_{\mathrm{d}}+f-\tau_{\mathrm{d}}-\alpha \Psi_{\mathrm{s}_{1}}^{\mathrm{d}} \eta_{\mathrm{r}_{2}}+\alpha \Psi_{\mathrm{r}_{1}}^{\mathrm{d}} \eta_{\mathrm{s}_{2}} \\
-\alpha \Psi_{r_{2}}^{\mathrm{d}} \eta_{\mathrm{s}_{1}}+\alpha \eta_{\mathrm{s}_{1}} \eta_{\mathrm{r}_{2}}-\alpha \eta_{\mathrm{s}_{2}} \eta_{\mathrm{r}_{1}},
\end{gathered}
$$

where (24) and (25) were utilized. Similarly, the stator and rotor flux open-loop error dynamics are developed as fol- 
lows:

$$
\begin{aligned}
& {\left[\begin{array}{c}
\dot{\eta}_{\mathrm{s}_{1}} \\
\dot{\eta}_{\mathrm{s}_{2}}
\end{array}\right]=\left[\begin{array}{c}
\dot{\Psi}_{\mathrm{s}_{1}}^{\mathrm{d}} \\
0
\end{array}\right]+R_{\mathrm{s}} \kappa_{1}\left[\begin{array}{c}
\Psi_{\mathrm{s}_{1}}^{\mathrm{d}} \\
0
\end{array}\right]-R_{\mathrm{s}} \kappa_{1}\left[\begin{array}{c}
\eta_{\mathrm{s}_{1}} \\
\eta_{\mathrm{s}_{2}}
\end{array}\right]} \\
& +R_{\mathrm{s}} \kappa_{2}\left[\begin{array}{c}
\eta_{\mathrm{r}_{1}} \\
\eta_{\mathrm{r}_{2}}
\end{array}\right]-R_{\mathrm{s}} \kappa_{2}\left[\begin{array}{c}
\Psi_{\mathrm{r}_{1}}^{\mathrm{d}} \\
\Psi_{\mathrm{r}_{2}}^{\mathrm{d}}
\end{array}\right] \\
& +\left[\begin{array}{c}
0 \\
\Psi_{\mathrm{s}_{1}}^{\mathrm{d}}
\end{array}\right] \omega_{\mathrm{s}}+\left[\begin{array}{c}
\eta_{\mathrm{s}_{2}} \\
-\eta_{\mathrm{s}_{1}}
\end{array}\right] \omega_{\mathrm{s}}-\left[\begin{array}{c}
V_{\mathrm{s}_{1}} \\
V_{\mathrm{s}_{2}}
\end{array}\right], \\
& {\left[\begin{array}{c}
\dot{\eta}_{\mathrm{r}_{1}} \\
\dot{\eta}_{\mathrm{r}_{2}}
\end{array}\right]=\left[\begin{array}{c}
\dot{\Psi}_{\mathrm{r}_{1}}^{\mathrm{d}} \\
\dot{\Psi}_{\mathrm{r}_{2}}^{\mathrm{d}}
\end{array}\right]+R_{\mathrm{r}} \kappa_{2}\left[\begin{array}{c}
\eta_{\mathrm{s}_{1}} \\
\eta_{\mathrm{s}_{2}}
\end{array}\right]-R_{\mathrm{r}} \kappa_{2}\left[\begin{array}{c}
\Psi_{\mathrm{s}_{1}}^{\mathrm{d}} \\
0
\end{array}\right]} \\
& +R_{\mathrm{r}} \kappa_{1}\left[\begin{array}{c}
\Psi_{\mathrm{r}_{1}}^{\mathrm{d}} \\
\Psi_{\mathrm{r}_{2}}^{\mathrm{d}}
\end{array}\right]-R_{\mathrm{r}} \kappa_{1}\left[\begin{array}{c}
\eta_{\mathrm{r}_{1}} \\
\eta_{\mathrm{r}_{2}}
\end{array}\right] \\
& +n_{\mathrm{p}} \omega\left[\begin{array}{c}
-\eta_{\mathrm{r}_{2}} \\
\eta_{\mathrm{r}_{1}}
\end{array}\right]-n_{\mathrm{p}} \omega\left[\begin{array}{c}
-\Psi_{\mathrm{r}_{1}}^{\mathrm{d}} \\
\Psi_{\mathrm{r}_{2}}^{\mathrm{d}}
\end{array}\right] \\
& +\left[\begin{array}{c}
-\Psi_{\mathrm{r}_{2}}^{\mathrm{d}} \\
\Psi_{\mathrm{r}_{1}}^{\mathrm{d}}
\end{array}\right] \omega_{\mathrm{s}}-\left[\begin{array}{c}
-\eta_{\mathrm{r}_{2}} \\
\eta_{\mathrm{r}_{1}}
\end{array}\right] \omega_{\mathrm{s}}-\left[\begin{array}{c}
V_{\mathrm{r}_{1}} \\
V_{\mathrm{r}_{2}}
\end{array}\right],
\end{aligned}
$$

where (16) and (17) were utilized.

\subsection{Controller formulation}

The control inputs are designed based on the subsequent stability analysis presented in Appendices A2 and A4. The desired torque trajectory, $\tau_{\mathrm{d}}(t)$, is designed as

$$
\tau_{\mathrm{d}}=K e+\frac{\rho_{\mathrm{z}}^{2} e}{\varepsilon}+J \dot{\omega}_{\mathrm{d}}-\hat{f}_{\mathrm{s}},
$$

where $\hat{f}_{\mathrm{s}}(\cdot) \triangleq \frac{1}{\sigma s+1} \operatorname{sat}\{\hat{f}(\cdot)\}, \operatorname{sat}\{\cdot\}$ is the saturation function, $\hat{f}(\cdot) \in \mathbb{R}$ is the estimate of $f(\cdot)$ which is designed in Section 4, $s$ is the Laplace variable, $\varepsilon$ and $\sigma \in \mathbb{R}^{+}$ are constants, $K \in \mathbb{R}^{+}$is a control gain, and $e(t)$ and $\rho_{\mathrm{z}}(\omega)$ were previously introduced in (23) and Remark 2 , respectively.

Remark 4 Since $\frac{1}{\sigma s+1}$ is a proper bounded filter and the output of the saturation function is always bounded; hence, it can be concluded that $\hat{f}_{\mathrm{s}}(\cdot)$ and $\dot{\hat{f}}_{\mathrm{s}}(\cdot)$ are bounded. Thus, it may be concluded that $\left|\hat{f}_{\mathrm{S}}(\cdot)\right| \leqslant \rho_{\mathrm{S}}$ where $\rho_{\mathrm{s}} \in \mathbb{R}^{+}$is a bounding constant.

Remark 5 The desired stator flux, $\Psi_{\mathrm{S}_{1}}^{\mathrm{d}}(t)$, is designed such that

i) $\Psi_{\mathrm{s}_{1}}^{\mathrm{d}}(t)>0$

ii) $\Psi_{\mathrm{s}_{1}}^{\mathrm{d}}(t), \dot{\Psi}_{\mathrm{s}_{1}}^{\mathrm{d}}(t)$, and $\ddot{\Psi}_{\mathrm{s}_{1}}^{\mathrm{d}}(t)$ are bounded, and

iii) power loss in the system is minimized. Refer to Appendix A1 for details. as

The first entry of the desired rotor flux, $\Psi_{\mathrm{r}_{1}}^{\mathrm{d}}(t)$, is designed

$$
\begin{aligned}
\Psi_{\mathrm{r}_{1}}^{\mathrm{d}}= & \frac{1}{R_{\mathrm{s}} \kappa_{2}}\left[\dot{\Psi}_{\mathrm{s}_{1}}^{\mathrm{d}}+R_{\mathrm{s}} \kappa_{1} \Psi_{\mathrm{s}_{1}}^{\mathrm{d}}-\alpha \Psi_{\mathrm{r}_{2}}^{\mathrm{d}} e-R_{\mathrm{s}} \kappa_{1} \eta_{\mathrm{s}_{1}}\right. \\
& \left.+\kappa_{\mathrm{s}_{1}} \eta_{\mathrm{s}_{1}}-V_{\mathrm{s}_{1}}\right]
\end{aligned}
$$

where $\kappa_{\mathrm{S}_{1}} \in \mathbb{R}^{+}$is a control gain.

Remark 6 To ensure the definition in $(22), \Psi_{\mathrm{r}_{2}}^{\mathrm{d}}(t)$ is designed such that $\Psi_{\mathrm{r}_{2}}^{\mathrm{d}}=\frac{-\tau_{\mathrm{d}}}{\alpha \Psi_{\mathrm{s}_{1}}^{\mathrm{d}}}$.
The auxiliary control input, $\omega_{\mathrm{s}}(t)$, is designed as

$$
\begin{aligned}
\omega_{\mathrm{s}}= & \frac{1}{\Psi_{\mathrm{s}_{1}}^{\mathrm{d}}}\left[R_{\mathrm{s}} \kappa_{2} \Psi_{r_{2}}^{\mathrm{d}}-\alpha \Psi_{r_{1}}^{\mathrm{d}} e+R_{\mathrm{s}} \kappa_{1} \eta_{s_{2}}\right. \\
& \left.-\kappa_{\mathrm{s}_{2}} \eta_{\mathrm{s}_{2}}+V_{\mathrm{s}_{2}}\right],
\end{aligned}
$$

where $\kappa_{\mathrm{S}_{2}} \in \mathbb{R}^{+}$is a control gain. The voltage control input, $V_{\mathrm{r}}(t)$, is designed as

$$
\begin{aligned}
V_{\mathrm{r}_{1}}= & \frac{\ddot{\Psi}_{\mathrm{s}_{1}}^{\mathrm{d}}}{R_{\mathrm{s}} \kappa_{2}}+\Theta_{1} \dot{\Psi}_{\mathrm{s}_{1}}^{\mathrm{d}}-\Theta_{2} \Psi_{\mathrm{s}_{1}}^{\mathrm{d}}+\Theta_{3} \Psi_{\mathrm{r}_{1}}^{\mathrm{d}}+\Theta_{4} \Psi_{\mathrm{r}_{2}}^{\mathrm{d}} \\
& +\left(\Theta_{5}-\Theta_{6} e+\Theta_{5} e^{2}\right) e+\left(\Theta_{8}-\Theta_{9} \Psi_{\mathrm{r}_{2}}^{\mathrm{d}} e\right) \eta_{\mathrm{s}_{1}} \\
& -\left(\Theta_{10}+\Theta_{11} e\right) \eta_{\mathrm{s}_{2}}+\left(\Theta_{12}-\Theta_{9} e\right) \eta_{\mathrm{s}_{2}} \eta_{\mathrm{r}_{1}} \\
& +\Theta_{13} \eta_{\mathrm{r}_{1}}+\frac{R_{\mathrm{s}} \kappa_{1}-\kappa_{\mathrm{s}_{1}}}{R_{\mathrm{s}} \kappa_{2}} V_{\mathrm{s}_{1}} \\
V_{\mathrm{r}_{2}}= & \Omega_{1}-\Omega_{2} \hat{f}_{\mathrm{s}}-\Omega_{3} e+\alpha e \eta_{\mathrm{s}_{1}}+\Omega_{4} \Psi_{\mathrm{r}_{2}}^{\mathrm{d}} \eta_{\mathrm{s}_{1}} \\
& +\Omega_{5} \eta_{\mathrm{s}_{2}}+\frac{\alpha^{2} \Psi_{\mathrm{s}_{1}}^{\mathrm{d}} \Psi_{\mathrm{r}_{2}}^{\mathrm{d}}}{R_{\mathrm{s}_{\mathrm{s}}} \kappa_{2} J} \eta_{\mathrm{r}_{1}} \\
& -\left(\Omega_{6}-\Omega_{7} \eta_{\mathrm{s}_{1}}\right) e \eta_{\mathrm{r}_{1}}-\frac{\alpha^{2} \Psi_{\mathrm{r}_{2}}^{\mathrm{d}}}{R_{\mathrm{s}_{\mathrm{s}}} \kappa_{2} J} \eta_{\mathrm{s}_{1}} \eta_{\mathrm{r}_{1}} \\
& +\Omega_{8} \eta_{\mathrm{s}_{2}} \eta_{\mathrm{r}_{1}}-\Omega_{8} \eta_{\mathrm{s}_{1}} \eta_{\mathrm{r}_{2}}+\Omega_{9} \eta_{\mathrm{r}_{2}}-\Omega_{10} e^{2}
\end{aligned}
$$

where the terms $\Theta_{i}(t)$ and $\Omega_{j}(t)$ for $i=1, \ldots, 13$ and $j=1, \ldots, 10$ are explicitly defined in Appendix A6.

\subsection{Stability analysis}

Theorem 1 Given the error system dynamics in (28)(30) and the designed terms in (31)-(35) along with Remark 6, the tracking error signals given in (23)-(25) are GUUB and all signals remain bounded under closed-loop operation.

Proof See Appendix A2.

\section{Estimation of uncertain system nonlineari- ties}

The control objective is to maximize the aerodynamic rotor power, $P_{\text {aero }}(t)$, captured by a variable speed wind turbine with structurally uncertain system nonlinearities, $f(\cdot)$, by controlling the rotor speed, $\omega(t)$. The control development in Section 3 requires estimating the uncertain nonlinearities, $f(\cdot)$. The estimate of $f(\cdot)$, denoted by $\hat{f}(\cdot)$, is developed for two reasons: i) The estimate $\hat{f}(\cdot)$ is used as a feed-forward term in the control design through $\hat{f}_{\mathrm{s}}(\cdot)$, and ii) since $P_{\text {aero }}(t)$ is unmeasurable, an estimate of the captured power, denoted by $\hat{P}_{\text {aero }}(t) \in \mathbb{R}$, is designed such that $\hat{P}_{\text {aero }}=-\hat{f} \omega$, and is used in the online planning of $\omega_{\mathrm{d}}(t)$.

\subsection{Observer error system}

The main objective of the observer is to estimate the uncertain system nonlinearities, $f(\cdot)$, such that $\hat{f}(\cdot) \rightarrow f(\cdot)$ as $t \rightarrow \infty$. To facilitate the observer design, the following system model is developed:

$$
J \dot{\hat{\omega}}=\tau_{\text {em }}-\hat{f},
$$

where $\hat{\omega}(t) \in \mathbb{R}^{+}$denotes the estimated rotor speed. The observer errors, denoted by $\tilde{\omega}(t), \tilde{f}(t) \in \mathbb{R}$, are defined as

$$
\begin{gathered}
\tilde{\omega} \triangleq \hat{\omega}-\omega, \\
\tilde{f} \triangleq \hat{f}-f .
\end{gathered}
$$

In addition, a filtered rotor speed estimation error, denoted by $r(t) \in \mathbb{R}$, is defined to facilitate the subsequent design 
and analysis as

$$
r \triangleq \dot{\tilde{\omega}}+k \tilde{\omega}
$$

where $k \in \mathbb{R}^{+}$is a control gain. After taking the first time derivative of (39) and premultiplying by $J$, the following expression can be obtained:

$$
J \dot{r}=-\dot{\hat{f}}+\dot{f}+\Phi-\tilde{\omega},
$$

where $\Phi(t) \in \mathbb{R}$ is defined as $\Phi \triangleq k J \dot{\tilde{\omega}}+\tilde{\omega}$ and (36)-(38) were utilized.

Remark 7 The mean value theorem can be utilized to upper bound $\Phi(t)$ such that $|\Phi(t)| \leqslant \rho_{\mathrm{N}}\|X\|$ where $X(t)=[\tilde{\omega}(t) r(t)]^{\mathrm{T}} \in \mathbb{R}^{2}$, and $\rho_{\mathrm{N}} \in \mathbb{R}^{+}$is a bounding constant [30].

\subsection{Observer design}

Based on the structure of (40), as well as the subsequent stability analysis, a continuous estimator law is proposed to achieve the stated estimator objectives where

$$
\dot{\hat{f}}=\left(k_{\mathrm{f}}+k\right) r+\rho_{0} \operatorname{sgn} \tilde{\omega},
$$

where $k_{\mathrm{f}} \in \mathbb{R}^{+}$is a control gain, $\rho_{0} \in \mathbb{R}^{+}$is a bounding constant, and $\operatorname{sgn}(\cdot) \in \mathbb{R}$ is the standard signum function.

\subsection{Stability analysis for nonlinear observer}

Theorem 2 The observer design in (41) ensures that asymptotic tracking is obtained in the sense that $\tilde{\omega}(t), \dot{\tilde{\omega}}(t)$, $r(t) \rightarrow 0$ and $\hat{f}(t) \rightarrow f(t)$ as $t \rightarrow \infty$.

Proof See Appendix A4.

\section{Trajectory generation and optimum seek- ing algorithm}

In Remark 3, it was assumed that a desired trajectory $\omega_{\mathrm{d}}(t)$ canbe designed such that $\omega_{\mathrm{d}}(t), \dot{\omega}_{\mathrm{d}}(t)$ and $\ddot{\omega}_{\mathrm{d}}(t)$ are bounded and $\omega_{\mathrm{d}}(t) \rightarrow \omega^{*}$, where $\omega^{*}$ is the unknown rotor speed that maximizes the aerodynamic rotor power, $P_{\text {aero }}(t)$, for a particular wind speed, $v(t)$, and blade pitch angle, $\beta$. As stated in Remark $1, P_{\text {aero }}(t)$ is unmeasurable, therefore the estimated captured power, $\hat{P}_{\text {aero }}(t)$, is used as the cost function to be optimized. The successive quadratic estimator (SQE) is selected as the optimum seeking algorithm. Although there are many optimization techniques in the literature [31-33], the advantage of this algorithm over conventional methods, such as the golden section search and simplex, is that no initial cost function values or bounds on the functional values are required [34]. The estimator approximates the cost function, $\hat{P}_{\text {aero }}(\hat{\omega}(t))$, as a quadratic function over a local bound and successively uses this property to predict the location of the optimum rotor speed, $\omega^{*}$.

To ensure that $\omega_{\mathrm{d}}(t), \dot{\omega}_{\mathrm{d}}(t)$ and $\ddot{\omega}_{\mathrm{d}}(t)$ are bounded, a filter-based form of the SQE is used, wherein at each iteration (new guess), $\omega_{\mathrm{d}}[n]$ is passed through a set of thirdorder stable and proper low pass filters to generate continuous bounded signals for $\omega_{\mathrm{d}}(t), \dot{\omega}_{\mathrm{d}}(t)$ and $\ddot{\omega}_{\mathrm{d}}(t)$. The following filters are used in this study:

$$
\begin{aligned}
& \omega_{\mathrm{d}}(t)=\frac{\zeta_{1}}{s^{3}+\zeta_{2} s^{2}+\zeta_{3} s+\zeta_{4}} \omega_{\mathrm{d}}[n], \\
& \dot{\omega}_{\mathrm{d}}(t)=\frac{\zeta_{1} s}{s^{3}+\zeta_{2} s^{2}+\zeta_{3} s+\zeta_{4}} \omega_{\mathrm{d}}[n],
\end{aligned}
$$

$$
\ddot{\omega}_{\mathrm{d}}(t)=\frac{\zeta_{1} s^{2}}{s^{3}+\zeta_{2} s^{2}+\zeta_{3} s+\zeta_{4}} \omega_{\mathrm{d}}[n],
$$

where $\zeta_{1}, \zeta_{2}, \zeta_{3}$, and $\zeta_{4} \in \mathbb{R}^{+}$are filter constants. The optimization algorithm waits until certain error thresholds are met before making the next guess (i.e., if $\left|\omega_{\mathrm{d}}(t)-\omega_{\mathrm{d}}[n]\right| \leqslant$ $\bar{e}_{1},|\tilde{f}(\cdot)| \leqslant \bar{e}_{2}$ and $\left|\omega(t)-\omega_{\mathrm{d}}(t)\right| \leqslant \bar{e}_{3}$, then $n=n+1$ where $\bar{e}_{1}, \bar{e}_{2}$, and $\bar{e}_{3} \in \mathbb{R}^{+}$are threshold constants, and $n \in \mathbb{Z}^{+}$).

\section{Numerical simulation}

A numerical case study is presented in this section to demonstrate the performance of the control strategy introduced in Section 3 and the numerical-based optimum seeking reference trajectory generator in Section 5 using MATLAB/Simulink. The plant model in (5) was assumed to correspond to a small wind turbine, possessing the following system nonlinearity

$$
f=-\frac{1}{2} \rho_{\mathrm{a}} A \frac{C_{\mathrm{p}}}{\omega} v_{\mathrm{a}}^{3} .
$$

For simulation purposes, a $350 \mathrm{~W}$ wind turbine extractor and a $0.5 \mathrm{hp}$ two-pole induction generator were selected. The model parameters and control gains are chosen as shown in Appendix A6.

The resulting rotor speed tracking error, $e(t)$, is shown in Fig. 1 while flux tracking errors, $\eta_{\mathrm{s}_{1}}(t), \eta_{\mathrm{s}_{2}}(t), \eta_{\mathrm{r}_{1}}(t)$, and $\eta_{\mathrm{r}_{2}}(t)$, are shown in Figs. 2 and 3, respectively. From these figures, it can be seen that globally uniformly bounded tracking errors have been achieved under the proposed control strategy. The voltage control inputs, $V_{\mathrm{r}_{1}}(t)$, and $V_{\mathrm{r}_{2}}(t)$, are shown in Fig. 4. The power coefficient function, $C_{\mathrm{p}}(\lambda)$ that is illustrated in Fig. 5 (a), is an example curve obtained using blade-element momentum theory [35]. It may be observed that $C_{\mathrm{p}}^{\max }=0.4405$ occurs when $\lambda^{*}=3.5$ which corresponds to $\omega^{*}=5.296$. The actual power efficiency measure, $C_{\mathrm{p}}(t)$, shown in Fig. $5(\mathrm{~b})$, indicates that $C_{\mathrm{p}}(t) \rightarrow 0.4401$ as $\omega(t) \rightarrow 5.3569$, which is illustrated in Fig. 6.

Additionally, the copper losses, $P_{\text {loss }}(t)$, desired stator flux, $\Psi_{\mathrm{s}_{1}}^{\mathrm{d}}(t)$, and estimator error, $\tilde{f}(t)$, are shown in Figs. 7, 8 and 9 , respectively. Overall, the simulation results demonstrate that the proposed control strategy performed satisfactorily and shows a robust response to structural uncertainties.

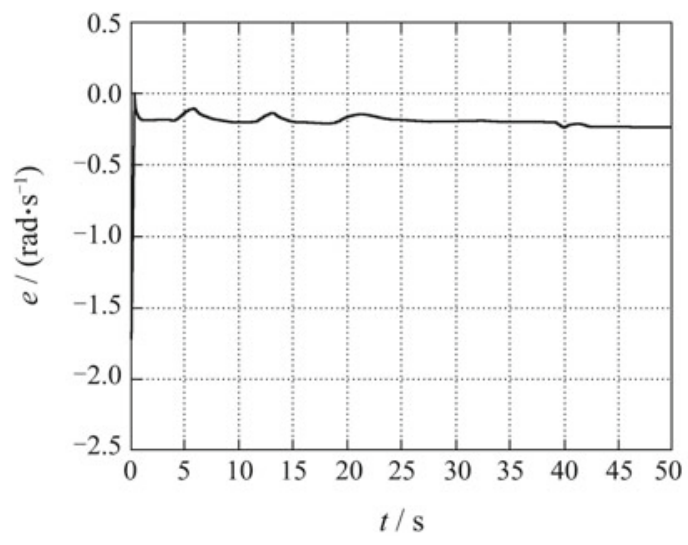

Fig. 1 Rotor speed tracking error $e(t)$. 


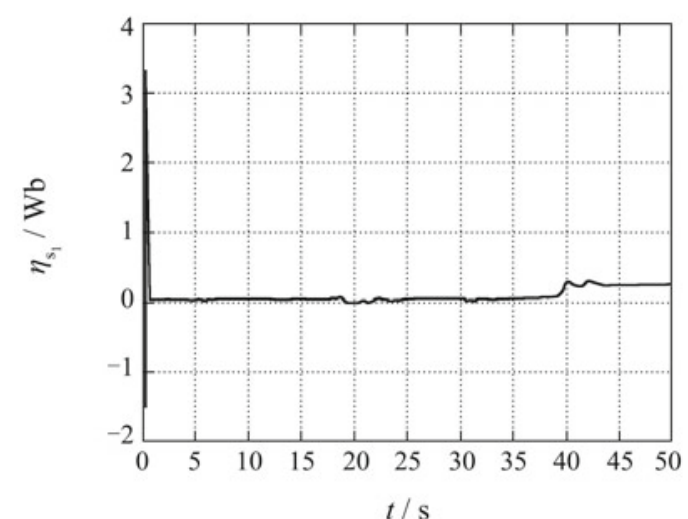

(a) $\eta_{\mathrm{s}_{1}}(t)$

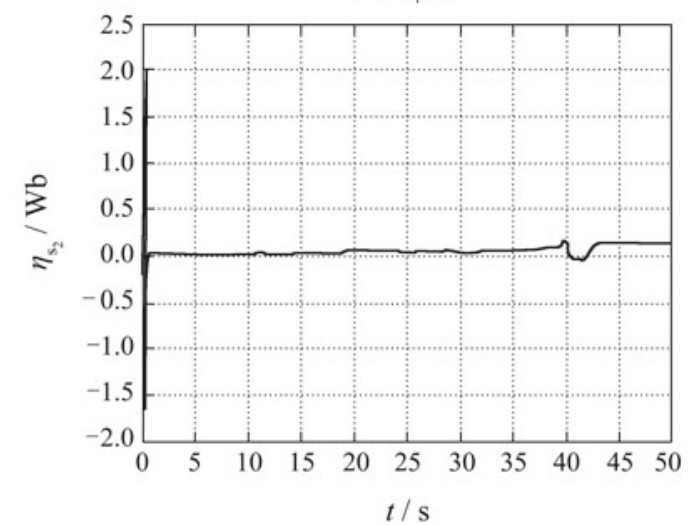

(b) $\eta_{\mathrm{s}_{2}}(t)$

Fig. 2 Stator flux tracking errors $\eta_{\mathrm{s}_{1}}(t)$ and $\eta_{\mathrm{s}_{2}}(t)$.

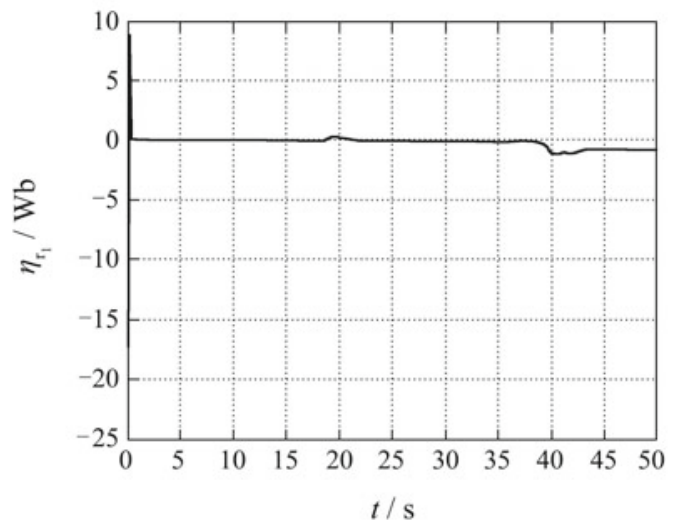

(a) $\eta_{r_{1}}(t)$

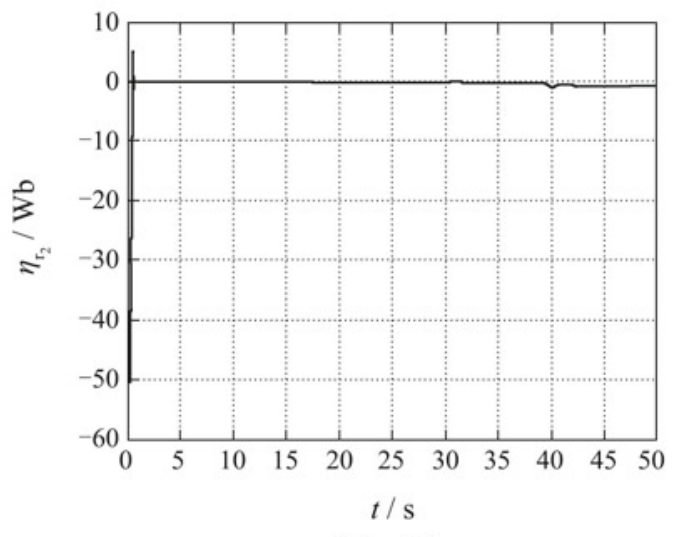

(b) $\eta_{\mathrm{F}_{2}}(t)$

Fig. 3 Rotor flux tracking errors $\eta_{\mathrm{r}_{1}}(t)$ and $\eta_{\mathrm{r}_{2}}(t)$.

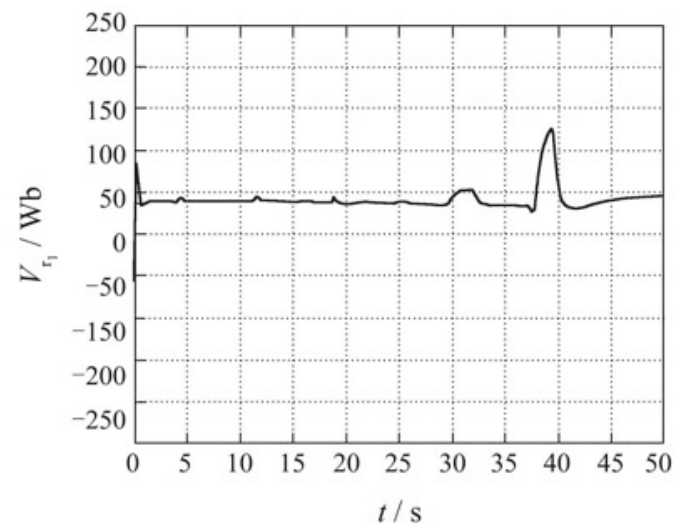

(a) $V_{\mathrm{r}_{1}}(t)$

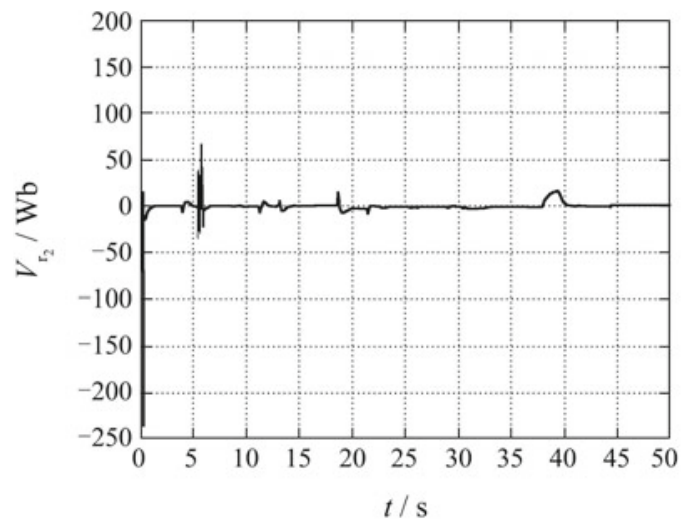

(b) $V_{\mathrm{r}_{2}}(t)$

Fig. 4 Voltage control inputs $V_{r_{1}}(t)$ and $V_{r_{2}}(t)$.

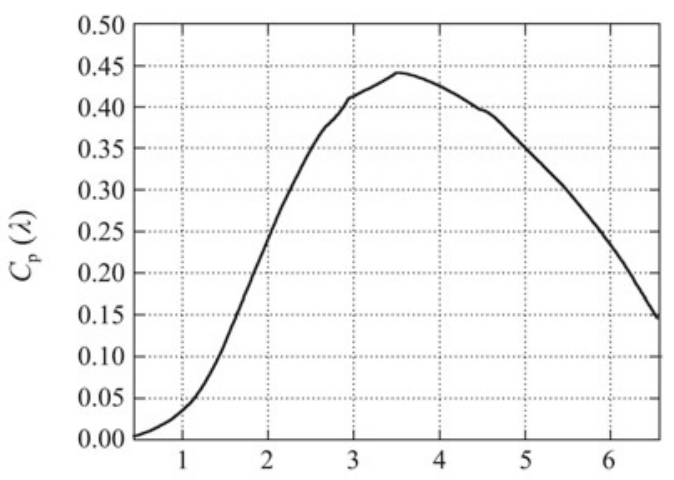

Tip speed ratio $\lambda$

(a) Simulated power coefficient function for wind turbine

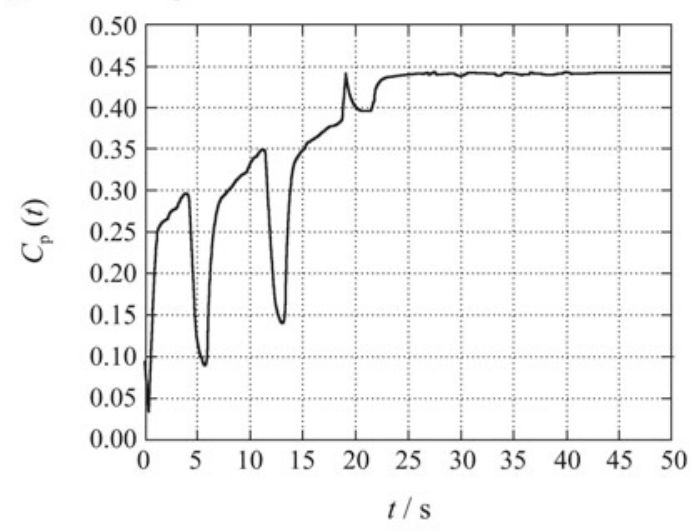

(b) Actual power coefficient function resulting from optimization algorithm

Fig. 5 Simulated and actual power coefficient functions. 


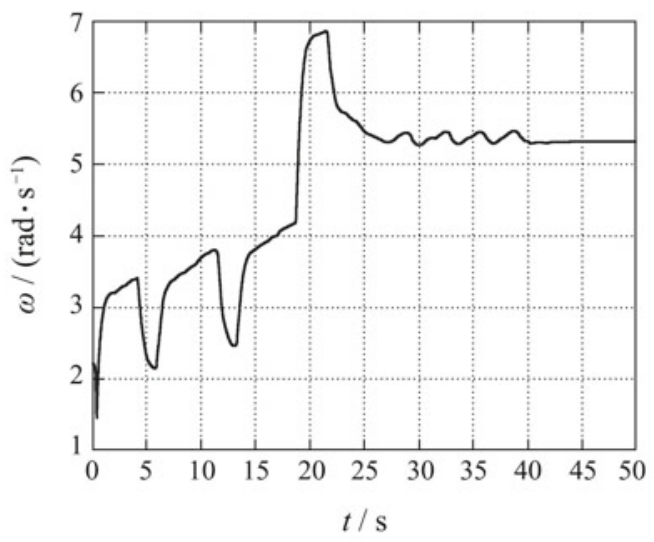

Fig. 6 Rotor speed $\omega(t)$ resulting from optimization algorithm.

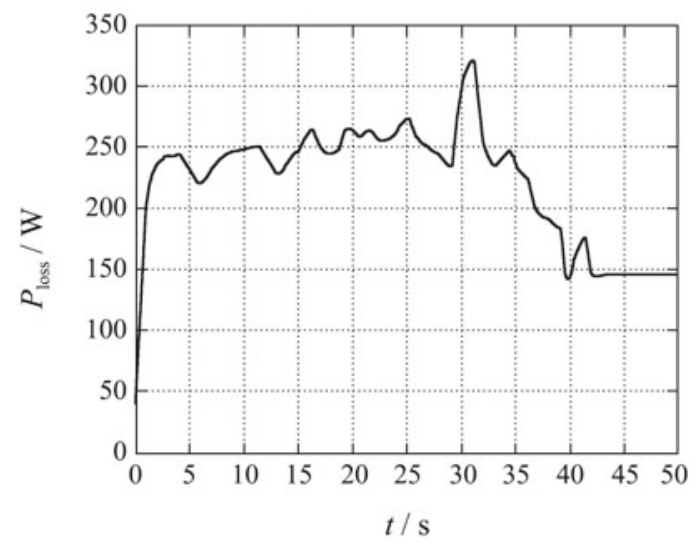

Fig. 7 Copper losses $P_{\text {loss }}(t)$ from optimization algorithm.

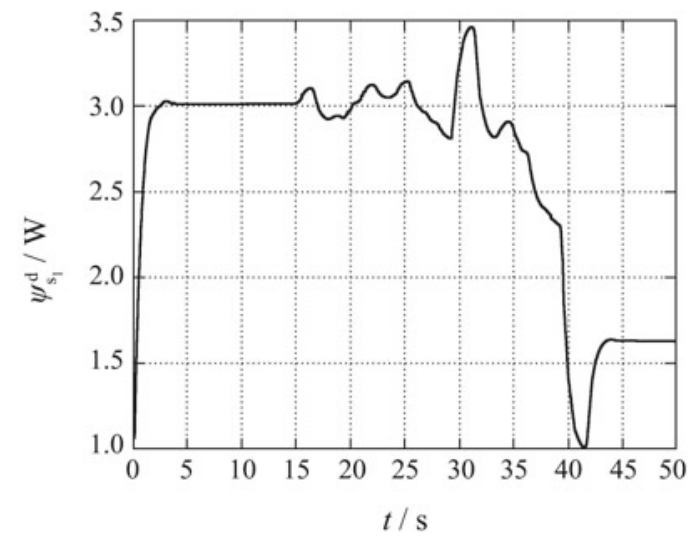

Fig. 8 Desired stator flux $\Psi_{\mathrm{s}_{1}}^{\mathrm{d}}(t)$.

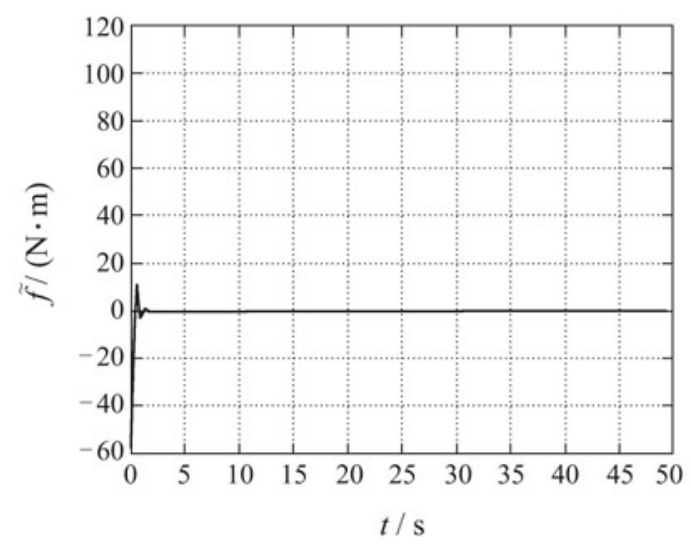

Fig. 9 Nonlinear observer error $\tilde{f}(t)$.

\section{Conclusions}

A nonlinear control strategy has been developed for a variable speed windturbine system with an internal induction generator to optimize the energy captured from the wind for a particular blade pitch angle. A desired rotor speed trajectory generator is presented that seeks the unknown optimal rotor speed while ensuring that the trajectory remains bounded and sufficiently differentiable. To track the desired trajectory, a robust tracking controller is developed. The proposed controller is proven to yield a globally uniformly ultimately bounded result while keeping the closedloop system stable via Lyapunov-based analysis. Simulation results were provided to verify the effectiveness of the control strategy. Future research will involve the implementation of the control strategy on a wind turbine extractor and eliminating the assumption of constant or slowly time varying wind speed.

\section{References}

[1] K. Johnson, L. Fingersh, M. Balas, et al. Methods for increasing region 2 power capture on a variable-speed wind turbine. ASME Journal of Solar Energy Engineering, 2004, 126(4): 1092 - 1100.

[2] U. Ozbay, E. Zergeroglu, S. Sivrioglu. Adaptive backstepping control of variable speed wind turbines. International Journal of Control, 2008, 81(6): 910 - 919.

[3] Y. Song, B. Dhinakaran, X. Bao. Variable speed control of wind turbines using nonlinear and adaptive algorithms. Journal of Wind Engineering and Industrial Aerodynamics, 2000, 85(3): 293 - 308.

[4] M. Hand, M. Balas. Non-linear and linear model based controller design for variable-speed wind turbines. The 3rd ASME/JSME Joint Fluids Engineering Conference. Golden, CO: National Renewable Energy Laboratory, 1999: 1 - 6.

[5] B. Liebst. Pitch control system for large-scale wind turbines. Journal of Energy, 1983, 7(2): 182 - 192.

[6] T. Knudsen, P. Andersen, S. Tiffner-Clausen. Comparing PI and robust control pitch controllers on a $400 \mathrm{KW}$ wind turbine by full scale tests. Proceedings of European Wind Conference. Dublin, 1997: 1 46.

[7] K. Stol, M. Balas. Full-state feedback control of a variable-speed wind turbine: a comparison of periodic and constant gains. Journal of Solar Energy Engineering, 2001, 123(4): 319 - 326.

[8] C. Tang, Y. Guo, J. Jiang. Nonlinear dual-mode control of variablespeed wind turbines with doubly fed induction generators. IEEE Transactions on Control Systems Technology, 2010, 19(4): 744 - 756.

[9] B. Boukhezzar, H. Siguerdidjane. Nonlinear control with wind estimation of a DFIG variable speed wind turbine for power capture optimization. Energy Conversion and Management, 2009, 50(4): 885 -892 .

[10] E. Iyasere, M. Salah, D. Dawson, et al. Nonlinear robust control to maximize energy capture in a variable speed wind turbine. Proceedings of the American Control Conference. New York: IEEE, 2008: 1824 - 1829.

[11] R. Chedid, S. Karaki, C. El-Chamali. Adaptive fuzzy control for wind-diesel weak power systems. IEEE Transactions on Energy Conversion, 2000, 15(1): $71-78$.

[12] X. Zhang, W. Wang, Y. Liu. Fuzzy control of variable speed wind turbine. Proceedings of the 6th World Congress on Intelligent Control and Automation. New York: IEEE, 2006: 3872 - 3876.

[13] A. Kusiak, W. Li, Z. Song. Dynamic control of wind turbines. Renewable Energy, 2010, 35(2): 456 - 463.

[14] W. Lin, C. M. Hong. Intelligent approach to maximum power point tracking control strategy for variable-speed wind turbine generation system. Energy, 2010, 35(6): 2440 - 2447. 
[15] A. Leon, M. Farias, P. Battaiotto, et al. Control strategy of a DVR to improve stability in wind farms using squirrel-cage induction generators. IEEE Transactions on Power Systems, 2011, 26(3): 1609 -1617 .

[16] T. Thiringer, J. Luomi. Comparison of reduced-order dynamic models of induction machines. IEEE Transactions on Power Systems, 2001, 16(1): $119-126$.

[17] A. Tapia, G. Tapia, J. Ostolaza, et al. Modeling and control of a wind turbine driven doubly fed induction generator. IEEE Transactions on Energy Conversion, 2003, 18(2): 194 - 204.

[18] A. Mullane, M. O'Malley. Inertial response of induction-machinebased wind turbines. IEEE Transactions on Power Systems, 2005, 20(3): $1495-1503$.

[19] J. Cathey, R. Calvin, A. Ayoub. Transient load model of an induction motor. IEEE Transactions on Power Apparatus and Systems, 1973, 92(4): 1399 - 1406.

[20] S. Muller, M Deicke. Doubly fed induction generator systems for wind turbines. IEEE Industry Application Magazine, 2002, 8(3): 26 -33 .

[21] J. Hu, D. Dawson. Adaptive control of induction motor systems despite rotor resistance uncertainty. Automatica, 1996, 32(8): 1127 -1143 .

[22] R. Datta, V. Ranganathan. A simple position-sensorless algorithm for rotor-side field-oriented-control of wound rotor induction machine. IEEE Transactions on Industrial Electronics, 2001 48(4): 786 - 793.

[23] R. Pena, J. Clare, G. Asher. Doubly fed induction generator using back to back PWM converters and its application to variablespeed wind-energy generation. IEE Proceedings - Electric Power Applications, 1996, 143(3): 231 - 241.

[24] R. Marino, S. Peresada, P. Valigi. Adaptive input-output linearizing control of induction motors. IEEE Transactions on Automatic Control, 1993, 38(2): $208-221$.

[25] R. Marino, S. Peresada, P. Tomei. Global adaptive output feedback control of induction motors with uncertain rotor resistance. IEEE Transactions on Automatic Control, 1999, 44(5): 967 - 983.

[26] K. Gopakumar, V. Ranganathan, S. Bhat. Vector control of induction motor with split phase stator windings. Proceedings of IEEE Industry Applications Society Annual Meeting. New York: IEEE, 1994: 569 574

[27] R. Datta, V. Ranganathan. Variable-speed wind power generation using doubly fed wound rotor induction machine - a comparison with alternative schemes. IEEE Transactions on Energy Conversion, 2002, 17(3): $414-421$

[28] R. Datta, V. Ranganathan. Direct power control of grid-connected wound rotor induction machine without rotor position sensors. IEEE Transactions on Power Electronics, 2001, 16(3): 390 - 399.

[29] P. Krause. Analysis of Electrical Machinery. New York: McGraw Hill, 1986.

[30] J. Slotine, W. Li. Applied Nonlinear Control. Englewood Cliffs: Prentice Hall, 1991.

[31] R. Rocha. A sensorless control for a variable speed wind turbine operating at partial load. Renewable Energy, 2011, 36(1): 132 - 141.

[32] A. Kusiak, H. Zheng. Optimization of wind turbine energy and power factor with an evolutionary computation algorithm. Energy, 2010, 35(3): $1324-1332$.

[33] R. Vepa. Nonlinear, optimal control of a wind turbine generator. IEEE Transactions on Energy Conversion, 2010, 26(2): 468 - 478.

[34] G. Reklaitis, A. Ravindran, K. Ragsdell. Engineering Optimization. New York: John Wiley \& Sons, 1983.

[35] M. Buhl. NWTC Design Codes (WT_Perf by Marshall Buhl). 2007: http://wind.nrel.gov/designcodes/simulators/wtperf/.

[36] P. Kokotovic. The joy of feedback: nonlinear and adaptive. IEEE Control Systems Magazine, 1992, 12(3): 7 - 17.

\section{Appendix}

A1 Online generating of $\Psi_{\mathrm{s}_{1}}^{\mathrm{d}}(t)$

The variable $\Psi_{\mathrm{s}_{1}}^{\mathrm{d}}(t)$ is designed to be a strictly positive function that ensures $\Psi_{\mathrm{s}_{1}}^{\mathrm{d}}(t), \dot{\Psi}_{\mathrm{s}_{1}}^{\mathrm{d}}(t)$, and $\ddot{\Psi}_{\mathrm{s}_{1}}^{\mathrm{d}}(t)$ are bounded with an optimum reduction in copper loss. The copper loss denoted by $P_{\text {loss }}(t)$, is defined as

$$
P_{\text {loss }} \triangleq P_{\text {in }}-P_{\text {out }}=I_{\mathrm{r}}^{\mathrm{T}} V_{\mathrm{r}}+\tau_{\mathrm{em}} \omega-I_{\mathrm{s}}^{\mathrm{T}} V_{\mathrm{s}} .
$$

Substituting (16)-(19) into (a1) results in

$$
\begin{aligned}
P_{\text {loss }}= & \left(\kappa_{1} \Psi_{\mathrm{r}}-\kappa_{2} \Psi_{\mathrm{s}}\right)^{\mathrm{T}}\left(\dot{\Psi}_{\mathrm{r}}+R_{\mathrm{r}} \kappa_{1} \Psi_{\mathrm{r}}-R_{\mathrm{r}} \kappa_{2} \Psi_{\mathrm{s}}+\Delta \Psi_{\mathrm{r}} \omega_{\mathrm{s}}\right. \\
& \left.-n_{p} \omega \Delta \Psi_{\mathrm{r}}\right)-\left(\kappa_{1} \Psi_{\mathrm{s}}-\kappa_{2} \Psi_{\mathrm{r}}\right)^{\mathrm{T}}\left(\dot{\Psi}_{\mathrm{s}}+R_{\mathrm{s}} \kappa_{1} \Psi_{\mathrm{s}}\right. \\
& \left.-R_{\mathrm{s}} \kappa_{2} \Psi_{\mathrm{r}}+\Delta \Psi_{\mathrm{s}} \omega_{\mathrm{s}}\right)+\tau_{\mathrm{em}} \omega .
\end{aligned}
$$

At steady state, all time derivatives equal to zero and $\Psi_{\mathrm{r}}(t) \rightarrow$ $\Psi_{\mathrm{r}}^{\mathrm{d}}(t), \Psi_{\mathrm{s}}(t) \rightarrow \Psi_{\mathrm{s}}^{\mathrm{d}}(t), \tau_{\mathrm{em}}(t) \rightarrow \tau_{\mathrm{d}}(t), \omega(t) \rightarrow \omega_{\mathrm{d}}(t)$ as $t \rightarrow \infty$ (all error signals go to zero) resulting in the following expressions:

$$
\begin{aligned}
P_{\text {loss }}= & \left(\kappa_{1} \Psi_{\mathrm{r}}^{\mathrm{d}}-\kappa_{2} \Psi_{\mathrm{s}}^{\mathrm{d}}\right)^{\mathrm{T}}\left(R_{\mathrm{r}} \kappa_{1} \Psi_{\mathrm{r}}^{\mathrm{d}}-R_{\mathrm{r}} \kappa_{2} \Psi_{\mathrm{s}}^{\mathrm{d}}+\Delta \Psi_{\mathrm{r}}^{\mathrm{d}} \omega_{\mathrm{s}}\right. \\
& \left.-n_{\mathrm{p}} \omega_{\mathrm{d}} \Delta \Psi_{\mathrm{r}}^{\mathrm{d}}\right)+\tau_{\mathrm{d}} \omega_{\mathrm{d}}-\left(\kappa_{1} \Psi_{\mathrm{s}}^{\mathrm{d}}-\kappa_{2} \Psi_{\mathrm{r}}^{\mathrm{d}}\right)^{\mathrm{T}} \\
& \cdot\left(R_{\mathrm{s}} \kappa_{1} \Psi_{\mathrm{s}}^{\mathrm{d}}-R_{\mathrm{s}} \kappa_{2} \Psi_{\mathrm{r}}^{\mathrm{d}}+\Delta \Psi_{\mathrm{s}}^{\mathrm{d}} \omega_{\mathrm{s}}\right) \\
\Psi_{\mathrm{r}_{1}}^{\mathrm{d}}= & \frac{\kappa_{1}}{\kappa_{2}} \Psi_{\mathrm{s}_{1}}^{\mathrm{d}}-\frac{V_{\mathrm{s}_{1}}}{R_{\mathrm{s}} \kappa_{2}} \\
\tau_{\mathrm{d}}= & -\hat{f}_{\mathrm{s}} \\
\Psi_{\mathrm{r}_{2}}^{\mathrm{d}}= & \frac{\hat{f}_{\mathrm{s}}}{\alpha \Psi_{\mathrm{s}_{1}}^{\mathrm{d}}} \\
\omega_{\mathrm{s}}= & \frac{R_{\mathrm{s}} \kappa_{2} \hat{f}_{\mathrm{s}}}{\alpha\left(\Psi_{\mathrm{s}_{1}}^{\mathrm{d}}\right)^{2}}+\frac{V_{\mathrm{s}_{2}}}{\Psi_{\mathrm{s}_{1}}^{\mathrm{d}}}
\end{aligned}
$$

where (29), (31), and Remark 6 were utilized. After substituting (a4)-(a7) into (a3), $P_{\text {loss }}$ can be expressed as a function of $\Psi_{\mathrm{s}_{1}}^{\mathrm{d}}(t)$ as

$$
\begin{aligned}
P_{\text {loss }}= & {\left[\frac{\kappa_{1}^{4} R_{\mathrm{r}}}{\kappa_{2}^{2}}+\kappa_{2}^{2} R_{\mathrm{r}}-2 \kappa_{1}^{2} R_{\mathrm{s}}\right]\left(\Psi_{\mathrm{s}_{1}}^{\mathrm{d}}\right)^{2}+\frac{2 \hat{f}_{\mathrm{s}}}{\alpha^{2} \kappa_{2}^{2} R_{\mathrm{s}}} } \\
& \cdot\left[\kappa_{1} V_{\mathrm{s}_{1}} \hat{f}_{\mathrm{s}}\left(\kappa_{1}^{2} R_{\mathrm{r}}+\kappa_{2}^{2} R_{\mathrm{s}}\right)+\kappa_{2} V_{\mathrm{s}_{2}}\left(\frac{2 \alpha \kappa_{1}^{2} R_{\mathrm{r}} V_{\mathrm{s}_{1}}^{2}}{R_{\mathrm{s}}}\right.\right. \\
& \left.\left.-\kappa_{2}^{3} R_{\mathrm{s}} n_{\mathrm{p}} \omega_{\mathrm{d}} \hat{f}_{\mathrm{s}}-\alpha \kappa_{2}^{2} R_{\mathrm{s}} \omega_{\mathrm{d}} \hat{f}_{\mathrm{s}}-\alpha \kappa_{2}^{2} V_{\mathrm{s}_{1}}^{2}\right)\right] \frac{1}{\Psi_{\mathrm{s}_{1}}^{\mathrm{d}}} .
\end{aligned}
$$

The expression in (a8) is then used as cost function in a filter-based SQE numerical minimization algorithm, similar to Section 5, with $\Psi_{\mathrm{S}_{1}}^{\mathrm{d}}(t)$ as the functional value. This ensures an optimum reduction in copper losses and boundedness of $\Psi_{\mathrm{s}_{1}}^{\mathrm{d}}(t), \dot{\Psi}_{\mathrm{s}_{1}}^{\mathrm{d}}(t)$, and $\ddot{\Psi}_{\mathrm{s}_{1}}^{\mathrm{d}}(t)$.

\section{A2 Proof of Theorem 1}

A nonnegative function, denoted by $V(t) \in \mathbb{R}$, is defined as

$$
V=\frac{1}{2} J e^{2}+\frac{1}{2} \eta_{\mathrm{s}}^{\mathrm{T}} \eta_{\mathrm{s}}+\frac{1}{2} \eta_{\mathrm{r}}^{\mathrm{T}} \eta_{\mathrm{r}}
$$

which can be rewritten as

$$
V=\frac{1}{2} z^{\mathrm{T}} \operatorname{diag}\{J, 1,1,1,1\} z,
$$

and can thus be bounded using the Raleigh inequality as

$$
\lambda_{\min }\|z\|^{2} \leqslant V \leqslant \lambda_{\max }\|z\|^{2},
$$

where $z(t) \in \mathbb{R}^{5}$ is defined as $z \triangleq\left[\begin{array}{ll}e \eta_{\mathrm{s}}^{\mathrm{T}} & \eta_{\mathrm{r}}^{\mathrm{T}}\end{array}\right]^{\mathrm{T}}, \lambda_{\min } \triangleq$ $0.5 \min (J, 1)$, and $\lambda_{\max } \triangleq 0.5 \max (J, 1)$. Taking the time derivative of (a9) results in

$$
\dot{V}=e J \dot{e}+\left[\begin{array}{ll}
\eta_{\mathrm{s}_{1}} & \eta_{\mathrm{s}_{2}}
\end{array}\right]\left[\begin{array}{l}
\dot{\eta}_{\mathrm{s}_{1}} \\
\dot{\eta}_{\mathrm{s}_{2}}
\end{array}\right]+\left[\begin{array}{ll}
\eta_{\mathrm{r}_{1}} & \eta_{\mathrm{r}_{2}}
\end{array}\right]\left[\begin{array}{c}
\dot{\eta}_{\mathrm{r}_{1}} \\
\dot{\eta}_{\mathrm{r}_{2}}
\end{array}\right] .
$$

After substituting (28)-(30) into (a12), $\dot{V}(t)$ can be expressed as

$$
\begin{aligned}
\dot{V}= & e\left[J \dot{\omega}_{\mathrm{d}}+f-\tau_{\mathrm{d}}-\alpha \Psi_{\mathrm{s}_{1}}^{\mathrm{d}} \eta_{\mathrm{r}_{2}}+\alpha \Psi_{\mathrm{r}_{1}}^{\mathrm{d}} \eta_{\mathrm{s}_{2}}\right. \\
& \left.-\alpha \Psi_{\mathrm{r}_{2}}^{\mathrm{d}} \eta_{\mathrm{s}_{1}}+\alpha \eta_{\mathrm{s}_{1}} \eta_{\mathrm{r}_{2}}-\alpha \eta_{\mathrm{s}_{2}} \eta_{\mathrm{r}_{1}}\right] \\
& +\left[\eta_{\mathrm{s}_{1}} \eta_{\mathrm{s}_{2}}\right]\left\{\left[\begin{array}{c}
\Psi_{\mathrm{s}_{1}}^{\mathrm{d}} \\
0
\end{array}\right]+R_{\mathrm{s}_{1}} \kappa_{1}\left[\begin{array}{c}
\Psi_{\mathrm{s}_{1}}^{\mathrm{d}} \\
0
\end{array}\right]-R_{\mathrm{s}_{1}} \kappa_{1}\left[\begin{array}{c}
\eta_{\mathrm{s}_{1}} \\
\eta_{\mathrm{s}_{2}}
\end{array}\right]\right.
\end{aligned}
$$




$$
\begin{aligned}
& +R_{\mathrm{s}} \kappa_{2}\left[\begin{array}{c}
\eta_{\mathrm{r}_{1}} \\
\eta_{\mathrm{r}_{2}}
\end{array}\right]-R_{\mathrm{s}} \kappa_{2}\left[\begin{array}{c}
\Psi_{\mathrm{r}_{1}}^{\mathrm{d}} \\
\Psi_{\mathrm{r}_{2}}^{\mathrm{d}}
\end{array}\right]+\left[\begin{array}{c}
0 \\
\Psi_{\mathrm{s}_{1}}^{\mathrm{d}}
\end{array}\right] \omega_{\mathrm{s}} \\
& \left.+\left[\begin{array}{c}
\eta_{\mathrm{s}_{2}} \\
-\eta_{\mathrm{s}_{1}}
\end{array}\right] \omega_{\mathrm{s}}-V_{\mathrm{s}}\right\}+\left[\eta_{\mathrm{r}_{1}} \eta_{\mathrm{r}_{2}}\right]\left\{\left[\begin{array}{c}
\dot{\Psi}_{\mathrm{r}_{1}}^{\mathrm{d}} \\
\dot{\Psi}_{\mathrm{r}_{2}}^{\mathrm{d}}
\end{array}\right]\right. \\
& +R_{\mathrm{r}} \kappa_{2}\left[\begin{array}{c}
\eta_{\mathrm{s}_{1}} \\
\eta_{\mathrm{s}_{2}}
\end{array}\right]-R_{\mathrm{r}} \kappa_{2}\left[\begin{array}{c}
\Psi_{\mathrm{s}_{1}}^{\mathrm{d}} \\
0
\end{array}\right]+R_{\mathrm{r}} \kappa_{1}\left[\begin{array}{c}
\Psi_{\mathrm{r}_{1}}^{\mathrm{d}} \\
\Psi_{\mathrm{r}_{2}}^{\mathrm{d}}
\end{array}\right] \\
& \left.-R_{\mathrm{r}} \kappa_{1}\left[\begin{array}{c}
\eta_{\mathrm{r}_{1}} \\
\eta_{\mathrm{r}_{2}}
\end{array}\right]+\left[\begin{array}{c}
-\Psi_{\mathrm{r}_{2}}^{\mathrm{d}} \\
\Psi_{\mathrm{r}_{1}}^{\mathrm{d}}
\end{array}\right] \omega_{\mathrm{s}}-\left[\begin{array}{c}
V_{\mathrm{r}_{1}} \\
V_{\mathrm{r}_{2}}
\end{array}\right]\right\} .
\end{aligned}
$$

Substituting (31)-(35) as well as the first time derivative of $\Psi_{\mathrm{r}_{1}}^{\mathrm{d}}(t)$ and $\Psi_{\mathrm{r}_{2}}^{\mathrm{d}}(t)$, results in

$$
\begin{aligned}
\dot{V}= & e f+e \hat{f}_{\mathrm{s}}-K e^{2}-\kappa_{\mathrm{s}_{1}} \eta_{\mathrm{s}_{1}}^{2}-\kappa_{\mathrm{s}_{2}} \eta_{\mathrm{s}_{2}}^{2}-\kappa_{\mathrm{r}_{1}} \eta_{\mathrm{r}_{1}}^{2} \\
& -\kappa_{\mathrm{r}_{2}} \eta_{\mathrm{r}_{2}}^{2}-\frac{\rho_{\mathrm{z}}^{2} e^{2}}{\varepsilon}-\frac{\rho_{1}^{2} \eta_{\mathrm{r}_{1}}^{2}}{\varepsilon_{1}}-\frac{\rho_{2}^{2} \eta_{\mathrm{r}_{2}}^{2}}{\varepsilon_{2}} \\
& +\frac{f \eta_{\mathrm{r}_{1}}}{R_{\mathrm{s}} \kappa_{2} J \Psi_{\mathrm{s}_{1}}^{\mathrm{d}}}\left(\frac{2 e}{\varepsilon}\left(K \varepsilon-\rho_{\mathrm{z}}\left(e \partial \rho_{\mathrm{z}}-\rho_{\mathrm{z}}\right)\right)+J \dot{\omega}_{\mathrm{d}}\right. \\
& \left.+\hat{f}_{\mathrm{s}}\right)-\frac{f \eta_{\mathrm{r}_{2}}}{\alpha J \Psi_{\mathrm{s}_{1}}^{\mathrm{d}}}\left(\frac{1}{\varepsilon}\left(K \varepsilon-\rho_{\mathrm{z}}\left(2 e \partial \rho_{\mathrm{z}}-\rho_{\mathrm{z}}\right)\right)\right)
\end{aligned}
$$

where $\partial \rho_{\mathrm{z}}(\omega)$ denotes the partial derivative of $\rho_{\mathrm{z}}(\omega)$ with respect to $\omega$.

Remark 8 The functions $\rho_{1}(\cdot)$ and $\rho_{2}(\cdot)$ are designed in Appendix A3 such that

$$
\begin{aligned}
& \rho_{1} \geqslant \frac{\left|J \dot{\omega}_{\mathrm{d}}+\hat{f}_{\mathrm{s}}+2 K e-\frac{2 \rho_{\mathrm{z}} e\left(e \partial \rho_{\mathrm{z}}-\rho_{\mathrm{z}}\right)}{\varepsilon}\right|}{\left|R_{\mathrm{s}} \kappa_{2} J \Psi_{\mathrm{s}_{1}}^{\mathrm{d}}\right|}|f|, \\
& \rho_{2} \geqslant\left|\left(K-\frac{\rho_{\mathrm{z}}\left(2 \partial \rho_{\mathrm{z}}-\rho_{\mathrm{z}}\right)}{\varepsilon}\right) \frac{f}{\alpha J \Psi_{\mathrm{s}_{1}}^{\mathrm{d}}}\right| .
\end{aligned}
$$

From (a14), using Remarks 2 and 6 as well as definitions of $\rho_{1}(\cdot)$ and $\rho_{2}(\cdot)$, the function $\dot{V}(t)$ can be upper bounded as follows:

$$
\begin{aligned}
\dot{V} \leqslant & -K_{1} e^{2}-\kappa_{\mathrm{s}_{1}} \eta_{\mathrm{s}_{1}}^{2}-\kappa_{\mathrm{s}_{2}} \eta_{\mathrm{s}_{2}}^{2}-\kappa_{\mathrm{r}_{1}} \eta_{\mathrm{r}_{1}}^{2}-\kappa_{\mathrm{r}_{2}} \eta_{\mathrm{r}_{2}}^{2} \\
& +\left\{\rho_{\mathrm{s}}|e|-K_{2} e^{2}\right\}+\rho_{\mathrm{z}}|e|\left[1-\frac{\rho_{\mathrm{z}}|e|}{\varepsilon}\right] \\
& +\rho_{1}\left|\eta_{\mathrm{r}_{1}}\right|\left[1-\frac{\rho_{1}\left|\eta_{\mathrm{r}_{1}}\right|}{\varepsilon_{1}}\right]+\rho_{2}\left|\eta_{\mathrm{r}_{2}}\right|\left[1-\frac{\rho_{2}\left|\eta_{\mathrm{r}_{2}}\right|}{\varepsilon_{2}}\right],
\end{aligned}
$$

where the control gain, $K$, introduced in (31) is defined as $K \triangleq$ $K_{1}+K_{2}$ with $K_{1}, K_{2} \in \mathbb{R}^{+}$. Applying the nonlinear damping argument [36] to the curly bracketed terms on the right hand side of (a17) results in the following upper bound for $\dot{V}(t)$ :

$$
\dot{V} \leqslant-\gamma\|z\|^{2}+\bar{\varepsilon}
$$

where $\bar{\varepsilon}, \gamma \in \mathbb{R}^{+}$are defined as $\bar{\varepsilon} \triangleq \varepsilon+\varepsilon_{1}+\varepsilon_{2}+\frac{\rho_{\mathrm{s}}^{2}}{K_{2}}$ and $\gamma \triangleq \min \left(K_{1}, \kappa_{\mathrm{s}_{1}}, \kappa_{\mathrm{s}_{2}}, \kappa_{\mathrm{r}_{1}}, \kappa_{\mathrm{r}_{2}}\right)$. From (a11) and (a18), the following relationship can be obtained:

$$
\dot{V} \leqslant-\frac{\gamma V}{\lambda_{\max }}+\bar{\varepsilon} .
$$

From (a11) and (a19), the error signal $\|z(t)\|$ can be upper bounded as

$$
\|z(t)\| \leqslant \sqrt{\beta_{0} \exp \left(-\beta_{1} t\right)+\beta_{2}\left[1-\exp \left(-\beta_{1} t\right)\right]},
$$

where $\beta_{0} \triangleq \frac{\lambda_{\max }}{\lambda_{\min }}\left\|z\left(t_{0}\right)\right\|^{2}, \beta_{1} \triangleq \frac{\gamma}{\lambda_{\max }}$, and $\beta_{2} \triangleq \frac{\lambda_{\max } \bar{\varepsilon}}{\lambda_{\min } \gamma}$. From (a20), it can be shown that $e(t), \eta_{\mathrm{s}}(t), \eta_{\mathrm{r}}(t) \in \mathcal{L}_{\infty}$. Since $e(t) \in \mathcal{L}_{\infty},(23)$ can be used along with Remark 3, to show that $\omega(t) \in \mathcal{L}_{\infty}$. After utilizing the fact that $\omega(t) \in \mathcal{L}_{\infty}$, from Assumption 6, it is apparent that $f(\cdot) \in \mathcal{L}_{\infty}$. After using Remarks 3 and 6 and the fact that $e(t) \in \mathcal{L}_{\infty}$, along with (31), it can be shown that $\tau_{\mathrm{d}}(t) \in \mathcal{L}_{\infty}$. Remark 5 can be used along with the facts that $\tau_{\mathrm{d}}(t), \Psi_{\mathrm{s}_{1}}^{\mathrm{d}}(t) \in \mathcal{L}_{\infty}$ to show that $\Psi_{\mathrm{r}_{2}}^{\mathrm{d}}(t) \in \mathcal{L}_{\infty}$. The expression in (32) can be used along with the above boundedness statements to show that $\Psi_{\mathrm{r}_{1}}^{\mathrm{d}}(t) \in \mathcal{L}_{\infty}$. Since all the signals on the right-hand-side of (28) are bounded then it can be concluded that $\dot{e}(t)$ is also bounded. From the time derivative of (23), it is easy to see that $\dot{\omega}(t)$ is bounded; thus, from Assumption 6, it is clear that $\dot{f}(\cdot)$ is bounded. The mechanical subsystem dynamics in (5) can be utilized to show that $\tau_{\mathrm{em}}(t)$ is bounded. Above boundedness statements can be utilized along with (24), (25), and (33) to show that $\Psi_{\mathrm{s}}(t), \Psi_{\mathrm{r}}(t), \omega_{\mathrm{s}}(t) \in \mathcal{L}_{\infty}$. Since $\omega(t)$ and $\dot{\omega}(t)$ are bounded, it is clear that $\dot{\rho}_{z}(\cdot) \in \mathcal{L}_{\infty}$. Above boundedness statements can be used along with Assumption 4, Remarks 3, 4 and 6 to prove that all the terms in Appendices A2 and A3 are bounded; thus, from (34) and (35), it can be concluded that $V_{\mathrm{r}_{1}}(t), V_{\mathrm{r}_{2}}(t) \in \mathcal{L}_{\infty}$. After utilizing the fact that $\dot{\Psi}_{\mathrm{s}_{1}}^{\mathrm{d}}(t) \in \mathcal{L}_{\infty}$ along with the above boundedness statements, from (29), it is easy to see that $\dot{\eta}_{\mathrm{s}}(t) \in \mathcal{L}_{\infty}$. The time derivatives of (31) and (32) can be utilized to show that $\dot{\tau}_{\mathrm{d}}(t)$ and $\dot{\Psi}_{\mathrm{r}_{1}}^{\mathrm{d}}(t)$ are bounded. From the time derivative of the expression in Remark 5, $\dot{\Psi}_{\mathrm{r}_{2}}^{\mathrm{d}}(t)$ can be shown to be bounded. From (30), it can be concluded that $\dot{\eta}_{\mathrm{r}_{1}}(t), \dot{\eta}_{\mathrm{r}_{2}}(t) \in \mathcal{L}_{\infty}$. The fact that $\dot{\eta}_{\mathrm{s}}(t)$, $\dot{\eta}_{\mathrm{r}}(t) \in \mathcal{L}_{\infty}$ can be used along with the time derivatives of (24) and (25) to show that $\dot{\Psi}_{\mathrm{s}}(t)$ and $\dot{\Psi}_{\mathrm{r}}(t)$ are bounded; thus, from the time derivative of (20), it is clear that $\dot{\tau}_{\text {em }}(t)$ is bounded. After taking the time derivative of (5), it can concluded that $\ddot{\omega}(t) \in \mathcal{L}_{\infty}$; thus, from the second time derivative of (23), it is clear that $\ddot{e}(t) \in \mathcal{L}_{\infty}$ where Remark 3 is utilized. From Assumption 6, it may be concluded that $\ddot{f}(\cdot) \in \mathcal{L}_{\infty}$. The application of standard signal chasing arguments permits the conclusion that all signals in the closedloop system remain bounded.

\section{A3 Design of $\rho_{\mathrm{z}}(\cdot), \rho_{\mathbf{1}}(\cdot)$, and $\rho_{\mathbf{2}}(\cdot)$}

The functions $\rho_{\mathrm{z}}(\cdot), \rho_{1}(\cdot)$, and $\rho_{2}(\cdot)$ are designed to ensure that the conditions in Remarks 2 and 8 are met

$$
\begin{aligned}
\rho_{\mathrm{z}}= & \left|\frac{1}{2} \rho_{\mathrm{a}} A \frac{0.45}{\omega} v^{3}\right|, \\
\rho_{1}= & \left|\frac{\rho_{\mathrm{z}}}{R_{\mathrm{s}} \kappa_{2} J \Psi_{\mathrm{s}_{1}}^{\mathrm{d}}}\right|\left[\frac{2 \rho_{\mathrm{z}}\left|\partial \rho_{\mathrm{z}}\right| e^{2}}{\varepsilon}+\frac{2 \rho_{\mathrm{z}}^{2}|e|}{\varepsilon}+2 K|e|\right. \\
& \left.+J\left|\dot{\omega}_{\mathrm{d}}\right|+\left|\hat{f}_{\mathrm{s}}\right|\right], \\
\rho_{2}= & \left|\frac{\rho_{\mathrm{z}}}{\alpha J \Psi_{\mathrm{s}_{1}}^{\mathrm{d}}}\right|\left(K+\frac{\rho_{\mathrm{z}}+2 \rho_{\mathrm{z}}\left|\partial \rho_{\mathrm{z}} e\right|}{\varepsilon}\right) .
\end{aligned}
$$

\section{A4 Proof of Theorem 2}

Before presenting the stability analysis, the following lemma will be introduced and later invoked.

Lemma 1 Let the auxiliary function $L(t) \in \mathbb{R}$ be defined as

$$
L \triangleq r\left(\dot{f}-\rho_{0} \operatorname{sgn} \tilde{\omega}\right) .
$$

If the control gain $\rho_{0}$ is selected to satisfy the sufficient condition $\rho_{0}>|\dot{f}(\cdot)|+\frac{|\ddot{f}(\cdot)|}{k}$, then $\int_{t_{0}}^{t} L(\tau) \mathrm{d} \tau \leqslant \zeta$ where $\zeta \in \mathbb{R}^{+}$is defined as $\zeta \triangleq \rho_{\mathrm{o}}\left|\tilde{\omega}\left(t_{0}\right)\right|-\tilde{\omega}\left(t_{0}\right) \dot{f}\left(t_{0}\right)$.

Proof See Appendix A5.

Define an auxiliary function $P(t) \in \mathbb{R}$ as

$$
P \triangleq \zeta-\int_{t_{\mathrm{o}}}^{t} L(\tau) \mathrm{d} \tau,
$$

where $\zeta$ and $L(t)$ have been defined in Lemma 1. Based on the nonnegativity of $P(t)$ (see proof of Lemma 1), we define a nonnegative function $V_{\mathrm{o}}(t) \in \mathbb{R}$ as follows:

$$
V_{\mathrm{o}} \triangleq \frac{1}{2} \tilde{\omega}^{2}+\frac{1}{2} J r^{2}+P
$$

After taking the time derivative of (a26) and utilizing (40), (a24), and the time derivative of (a25), we can conveniently rearrange terms to obtain the following expression:

$$
\dot{V}_{\mathrm{o}}=-k \tilde{\omega}^{2}-r \dot{\hat{f}}+r \Phi+r \rho_{0} \operatorname{sgn} \tilde{\omega} .
$$

After substituting (41) and utilizing Remark 7, simple algebraic manipulations can be used to obtain the following upper bound for 
$\dot{V}_{\mathrm{o}}(t)$

$$
\dot{V}_{\mathrm{o}} \leqslant-k\|X\|^{2}+\left[|r| \rho_{\mathrm{N}}\|X\|-k_{\mathrm{f}}|r|^{2}\right] .
$$

Applying the nonlinear damping argument [25] to the bracketed term in (a28) results in the following upper bound for $\dot{V}_{\mathrm{o}}(t)$

$$
\dot{V}_{\mathrm{o}} \leqslant-\left[k-\frac{\rho_{\mathrm{N}}^{2}}{k_{\mathrm{f}}}\right]\|X\|^{2} .
$$

From (a29), it is possible to state that

$$
\dot{V}_{\mathrm{o}} \leqslant-\varsigma\|X\|^{2} \text { for } k_{\mathrm{f}}>\frac{\rho_{\mathrm{N}}^{2}}{k},
$$

where $\varsigma \in \mathbb{R}^{+}$is a constant. From (a30) and the analysis in this section, we can conclude that $X(t) \in \mathcal{L}_{\infty}$. From the definition of $X(t)$, it can be inferred that $\tilde{\omega}(t), \dot{\tilde{\omega}}(t), r(t) \in \mathcal{L}_{\infty}$. From (41), it is clear that $\dot{\hat{f}}(\cdot) \in \mathcal{L}_{\infty}$. Using standard signal chasing arguments, it can be shown that the all the signals in the closed-loop system remain bounded. In particular, from (40), it can be seen that $\dot{r}(t) \in \mathcal{L}_{\infty}$; thus, $\dot{X}(t) \in \mathcal{L}_{\infty}$. After employing a corollary to Barbalat's lemma [26], it is easy to show that $\|X(t)\| \rightarrow 0$ as $t \rightarrow \infty$. From the definition of $X(t)$, it can be concluded that $\tilde{\omega}(t), r(t) \rightarrow 0$ as $t \rightarrow \infty$. From (39), it is easy to see that $\dot{\tilde{\omega}}(t) \rightarrow 0$ as $t \rightarrow \infty$. From (36), the following relationship can be obtained

$$
J \dot{\tilde{\omega}}=f-\hat{f}=-\tilde{f} .
$$

From (a31), it is clear that $\dot{\tilde{\omega}}(t) \rightarrow 0$ implies that $|\tilde{f}(t)| \rightarrow 0$ thus $\hat{f}(t) \rightarrow f(t)$ so $\hat{P}_{\text {aero }}(t) \rightarrow P_{\text {aero }}(t)$ as $t \rightarrow \infty$.

\section{A5 Proof of Lemma 1}

Equation (39) can be substituted into (a24) and then integrated in time to obtain

$$
\begin{aligned}
\int_{t_{\mathrm{o}}}^{t} L(\tau) \mathrm{d} \tau= & \int_{t_{\mathrm{o}}}^{t} k \tilde{\omega}(\tau)\left(\dot{f}(\tau)-\rho_{0} \operatorname{sgn} \tilde{\omega}(\tau)\right) \mathrm{d} \tau \\
& +\int_{t_{\mathrm{o}}}^{t} \dot{\tilde{\omega}}(\tau) \dot{f}(\tau) \mathrm{d} \tau-\rho_{0} \int_{t_{\mathrm{o}}}^{t} \dot{\tilde{\omega}}(\tau) \operatorname{sgn} \tilde{\omega}(\tau) \mathrm{d} \tau
\end{aligned}
$$

The second integral in (a32) can be integrated by parts to obtain the following final expression:

$$
\begin{aligned}
\int_{t_{\mathrm{o}}}^{t} L(\tau) \mathrm{d} \tau= & \int_{t_{\mathrm{o}}}^{t} k \tilde{\omega}(\tau)\left(\dot{f}(\tau)-\frac{\ddot{f}(\tau)}{k}-\rho_{0} \operatorname{sgn} \tilde{\omega}(\tau)\right) \mathrm{d} \tau \\
& +\tilde{\omega}(t) \dot{f}(t)-\tilde{\omega}\left(t_{0}\right) \dot{f}\left(t_{0}\right) .
\end{aligned}
$$

An upper bound on the right hand side of (a33) can be written as

$$
\int_{t_{\mathrm{o}}}^{t} L(\tau) \mathrm{d} \tau=\int_{t_{\mathrm{o}}}^{t} k|\tilde{\omega}(\tau)|\left(|\dot{f}(\tau)|+\frac{|\ddot{f}(\tau)|}{k}-\rho_{0}\right) \mathrm{d} \tau .
$$

From (a34), it is clear that if $\rho_{0}>|\dot{f}(\cdot)|+\frac{|\ddot{f}(\cdot)|}{k}$, then Lemma 1 holds.

\section{A6 Definitions of $\Omega_{i}, \Theta_{j}$, and tables}

The terms $\Omega_{i}$ and $\Theta_{j}$ for $i=1, \ldots, 13$ and $j=1, \ldots, 10$, introduced in (34) and (35), respectively, are given by

$$
\begin{aligned}
\Theta_{1} \triangleq & \frac{\kappa_{\mathrm{s}_{1}}}{R_{\mathrm{s}} \kappa_{2}}, \Theta_{2} \triangleq \frac{R_{\mathrm{s}} \kappa_{1}^{2}-\kappa_{1} \kappa_{\mathrm{s}_{1}}+R_{\mathrm{s}} R_{\mathrm{r}} \kappa_{2}^{3}}{R_{\mathrm{s}} \kappa_{2}^{2}}, \\
\Theta_{3} \triangleq & \kappa_{1}\left(R_{\mathrm{s}}+R_{\mathrm{r}}\right)-\kappa_{\mathrm{s}_{1}}, \Theta_{4} \triangleq n_{\mathrm{p}} \omega-\omega_{\mathrm{s}}+\frac{\alpha \hat{f}_{\mathrm{s}}}{R_{\mathrm{s}} \kappa_{2} J}, \\
\Theta_{5} \triangleq & \frac{1}{R_{\mathrm{s}} \kappa_{2} \Psi_{\mathrm{s}_{1}}^{\mathrm{d}}}\left[\hat{f}_{\mathrm{s}}\left(\frac{\dot{\Psi}_{\mathrm{s}_{1}}^{\mathrm{d}}}{\Psi_{\mathrm{s}_{1}}^{\mathrm{d}}}+\frac{\Gamma}{J \varepsilon}\right)+\left(J \ddot{\omega}_{\mathrm{d}}-\dot{\hat{f}}_{\mathrm{s}}-\frac{J \dot{\omega}_{\mathrm{d}} \dot{\Psi}_{\mathrm{s}_{1}}^{\mathrm{d}}}{\Psi_{\mathrm{s}_{1}}^{\mathrm{d}}}\right.\right. \\
& \left.\left.+\frac{\alpha}{J}\left(K+\frac{\rho_{\mathrm{z}}^{2}}{\varepsilon}\right)\left(\Psi_{\mathrm{s}_{1}}^{\mathrm{d}}\right)^{2}\right)\right], \\
\Theta_{6} \triangleq & \frac{1}{\varepsilon R_{\mathrm{s}} \kappa_{2} \Psi_{\mathrm{s}_{1}}^{\mathrm{d}}}\left[\frac{K \varepsilon \dot{\Psi}_{\mathrm{s}_{1}}^{\mathrm{d}}+\rho_{\mathrm{z}}^{4}}{\Psi_{\mathrm{s}_{1}}^{\mathrm{d}}}+\frac{K^{2} \varepsilon+2 K \rho_{\mathrm{z}}^{2}-2 J \rho_{\mathrm{z}} \frac{\partial \rho_{\mathrm{z}}}{\partial \omega} \dot{\omega}_{\mathrm{d}}}{J}\right], \\
\Theta_{7} \triangleq & \frac{2 \rho_{\mathrm{z}} \frac{\partial \rho_{\mathrm{z}}}{\partial \omega}}{R_{\mathrm{s}} \kappa_{2} J \varepsilon^{2} \Psi_{\mathrm{s}_{1}}^{\mathrm{d}}}\left[K \varepsilon+\rho_{\mathrm{z}}^{2}\right],
\end{aligned}
$$

$$
\begin{aligned}
& \Theta_{8} \triangleq \kappa_{2}\left(R_{\mathrm{s}}+R_{\mathrm{r}}\right)+\frac{\kappa_{1}}{\kappa_{2}}\left(R_{\mathrm{s}} \kappa_{1}-\kappa_{\mathrm{s}_{1}}\right)+\frac{\left(\alpha \Psi_{\mathrm{r}_{2}}^{\mathrm{d}}\right)^{2}}{R_{\mathrm{s}} \kappa_{2} J}, \\
& \Theta_{9} \triangleq \frac{\alpha \Gamma}{R_{\mathrm{s}} \kappa_{2} J \varepsilon \Psi_{\mathrm{s} 1}^{\mathrm{d}}}, \\
& \Theta_{10} \triangleq \frac{1}{R_{\mathrm{s}} \kappa_{2} J}\left[\alpha^{2} \Psi_{\mathrm{r}_{1}}^{\mathrm{d}} \Psi_{\mathrm{r}_{2}}^{\mathrm{d}}+J \omega_{\mathrm{s}}\left(R_{\mathrm{s}} \kappa_{1}-\kappa_{\mathrm{s}_{1}}\right)\right], \\
& \Theta_{11} \triangleq \alpha-\frac{\alpha \Gamma \Psi_{\mathrm{r}_{1}}^{\mathrm{d}}}{R_{\mathrm{s}} \kappa_{2} J \varepsilon \Psi_{\mathrm{s}_{1}}^{\mathrm{d}}}, \quad \Theta_{12} \triangleq \frac{\alpha^{2} \Psi_{\mathrm{r}_{2}}^{\mathrm{d}}}{R_{\mathrm{s}} \kappa_{2} J}, \\
& \Theta_{13} \triangleq \kappa_{\mathrm{s}_{1}}+\kappa_{\mathrm{r}_{1}}-R_{\mathrm{s}} \kappa_{1}-R_{\mathrm{r}} \kappa_{1}+\frac{\rho_{1}^{2}}{\varepsilon_{1}}, \\
& \Omega_{1} \triangleq \frac{1}{\alpha \Psi_{\mathrm{s}_{1}}^{\mathrm{d}}}\left[\dot{\hat{f}}_{\mathrm{s}}-\left(J \ddot{\omega}_{\mathrm{d}}-\frac{J \dot{\omega}_{\mathrm{d}} \dot{\Psi}_{\mathrm{s}_{1}}^{\mathrm{d}}}{\Psi_{\mathrm{s}_{1}}^{\mathrm{d}}}-\alpha R_{\mathrm{r}} \kappa_{1} \Psi_{\mathrm{s}_{1}}^{\mathrm{d}} \Psi_{\mathrm{r}_{2}}^{\mathrm{d}}\right.\right. \\
& \left.\left.+\alpha \Psi_{\mathrm{s}_{1}}^{\mathrm{d}} \Psi_{\mathrm{r}_{1}}^{d}\left(n_{\mathrm{p}} \omega-\omega_{\mathrm{s}}\right)\right)\right] \text {, } \\
& \Omega_{2} \triangleq \frac{1}{\alpha J \varepsilon \Psi_{\mathrm{s}_{1}}^{\mathrm{d}}}\left[\frac{J \varepsilon \dot{\Psi}_{\mathrm{s}_{1}}^{\mathrm{d}}}{\Psi_{\mathrm{s}_{1}}^{\mathrm{d}}}+\Gamma\right] \\
& \Omega_{3} \triangleq \frac{1}{\alpha J \varepsilon^{2} \Psi_{\mathrm{s}_{1}}^{\mathrm{d}}}\left(\varepsilon \left(-\rho_{\mathrm{z}}^{2}\left(2 K+J \dot{\Psi}_{\mathrm{s}_{1}}^{\mathrm{d}}\right)+2 J \rho_{\mathrm{z}} \frac{\partial \rho_{\mathrm{z}}}{\partial \omega} \dot{\omega}_{\mathrm{d}}\right.\right. \\
& \left.\left.-K^{2} \varepsilon\right)-\rho_{\mathrm{z}}^{4}\right)+\alpha \Psi_{\mathrm{s}_{1}}^{\mathrm{d}}\left(1-\frac{K \dot{\Psi}_{\mathrm{s}_{1}}^{\mathrm{d}}}{\Psi_{\mathrm{s}_{1}}^{\mathrm{d}}}\right), \\
& \Omega_{4} \triangleq \frac{\Gamma}{J \varepsilon \Psi_{\mathrm{s}_{1}}^{\mathrm{d}}}, \quad \Omega_{5} \triangleq R_{\mathrm{r}}\left(\kappa_{1}+\kappa_{2}\right)+\Psi_{\mathrm{r}_{1}}^{\mathrm{d}} \Omega_{4}, \\
& \Omega_{6} \triangleq \frac{\alpha \Gamma}{R_{\mathrm{s}} \kappa_{2} J \varepsilon}, \quad \Omega_{7} \triangleq \frac{\Omega_{6}}{\Psi_{\mathrm{s}}^{\mathrm{d}}}, \quad \Omega_{8} \triangleq \frac{R_{\mathrm{s}} \kappa_{2}}{\alpha} \Omega_{6}, \\
& \Omega_{9} \triangleq \frac{\Gamma}{J \varepsilon}+\frac{\rho_{2}^{2}}{\varepsilon_{2}}+\kappa_{\mathrm{r}_{1}}-R_{\mathrm{r}} \kappa_{1}, \\
& \Omega_{10} \triangleq \frac{2 \rho_{\mathrm{z}} \frac{\partial \rho_{\mathrm{z}}}{\partial \omega}}{\alpha J \varepsilon^{2} \Psi_{\mathrm{s}_{1}}^{\mathrm{d}}}\left(K \varepsilon+\rho_{\mathrm{z}}^{2}\right),
\end{aligned}
$$

where $\Gamma \triangleq \rho_{\mathrm{z}}^{2}-2 \rho_{\mathrm{z}} \frac{\partial \rho_{\mathrm{z}}}{\partial \omega} e+K \varepsilon$ with $\kappa_{\mathrm{r}_{1}}, \kappa_{\mathrm{r}_{2}} \in \mathbb{R}^{+}$are control gains, $\varepsilon_{1}, \varepsilon_{2} \in \mathbb{R}^{+}$are constants, and $\rho_{1}(\cdot), \rho_{2}(\cdot) \in \mathbb{R}^{+}$are known functions designed in Appendix A2.

Table 1 Nomenclature.

\begin{tabular}{llc}
\hline$A$ & Rotor swept area & $\mathrm{m}^{2}$ \\
$C_{\mathrm{p}}$ & Power coefficient & - \\
$e$ & Rotor speed error & $\mathrm{rad} \cdot \mathrm{s}^{-1}$ \\
$f$ & System nonlinearities & $\mathrm{N} \cdot \mathrm{m}$ \\
$I, \bar{I}$ & Current & $\mathrm{A}$ \\
$J$ & Moment of inertia & $\mathrm{kg} \cdot \mathrm{m}^{2}$ \\
$k$ & Positive control gain & - \\
$k_{\mathrm{f}}$ & Positive control gain & - \\
$k_{1}$ & Positive constant & - \\
$k_{2}$ & Positive constant & - \\
$K$ & Positive control gain & - \\
$L$ & Inductance & $\mathrm{H}$ \\
$M$ & Mutual inductance & $\mathrm{H}$ \\
$n_{\mathrm{p}}$ & Number of generator pole pairs & - \\
$P_{\mathrm{aero}}$ & Aerodynamic power & $\mathrm{W}$ \\
$R$ & Resistance & $\Omega$ \\
$R_{\mathrm{b}}$ & Blade length & $\mathrm{m}$ \\
$v_{\mathrm{a}}$ & Wind speed & $\mathrm{m} \cdot \mathrm{s}^{-2}$ \\
$V, \bar{V}$ & Voltage & $\mathrm{V}$ \\
$\alpha$ & Positive constant & - \\
$\beta$ & Blade pitch angle & $\mathrm{rad}$ \\
$\Delta$ & Positive definite matrix & - \\
$\varepsilon$ & Positive constant & - \\
& &
\end{tabular}




\begin{tabular}{llc}
$\varepsilon_{1}$ & Positive constant & - \\
$\varepsilon_{2}$ & Positive constant & - \\
$\eta$ & Flux error & $\mathrm{Wb}$ \\
$\lambda$ & Tip-speed ratio & - \\
$\rho_{\mathrm{a}}$ & Air density & $\mathrm{kg} \cdot \mathrm{m}^{-3}$ \\
$\rho_{\mathrm{N}}$ & Bounding known constant & - \\
$\rho_{\mathrm{o}}$ & Bounding known constant & - \\
$\rho_{\mathrm{s}}$ & Bounding known constant & - \\
$\rho_{\mathrm{z}}$ & Bounding known function & - \\
$\sigma$ & Positive constant & - \\
$\tau_{\mathrm{aero}}$ & Aerodynamic torque & $\mathrm{N} \cdot \mathrm{m}$ \\
$\tau_{\mathrm{em}}$ & Electromagnetic torque & $\mathrm{N} \cdot \mathrm{m}$ \\
$\Psi, \Psi$ & Flux linkage & $\mathrm{Wb}$ \\
$\omega$ & Rotor speed & $\mathrm{rad} \cdot \mathrm{s}$ \\
$\omega_{\mathrm{s}}$ & Auxiliary control input & - \\
\hline
\end{tabular}

Table 2 Subscripts and superscripts.

\begin{tabular}{clcl}
\hline$*$ & Optimal value & $\max$ & Maximum value \\
$\mathrm{a}, \mathrm{b}$ & Frame component & $\mathrm{r}$ & Rotor \\
$\mathrm{d}$ & Desired value & $\mathrm{s}$ & Stator \\
\hline
\end{tabular}

Table 3 Values of the system parameters and control gains used in the numerical simulation.

\begin{tabular}{ccc}
\hline Symbol & Value & Unit \\
\hline$J$ & 2.4 & $\mathrm{Kg} \cdot \mathrm{m}^{2}$ \\
$k$ & 10 & - \\
$k_{\mathrm{f}}$ & 10 & - \\
$\kappa_{\mathrm{r}_{1}}$ & 1 & - \\
$\kappa_{\mathrm{r}_{2}}$ & 1 & - \\
$\kappa_{\mathrm{s}_{1}}$ & 50 & - \\
$\kappa_{\mathrm{s}_{2}}$ & 50 & - \\
$K$ & 50 & - \\
$L_{\mathrm{s}}$ & 0.078 & $\mathrm{H}$ \\
$M$ & 0.571 & $\mathrm{H}$ \\
$n_{\mathrm{p}}$ & 1 & - \\
$R_{\mathrm{b}}$ & 1.52 & $\mathrm{~m}$ \\
$R_{\mathrm{r}}$ & 7.25 & $\Omega$ \\
$R_{\mathrm{s}}$ & 5.55 & $\Omega$ \\
$v_{\mathrm{a}}$ & 2.3 & $\mathrm{~m} \cdot \mathrm{s}^{-2}$ \\
$\beta$ & 2.4 & $\mathrm{rad}$ \\
$\varepsilon$ & 100 & - \\
$\varepsilon_{1}$ & 1 & - \\
$\varepsilon_{2}$ & 1 & - \\
$\rho_{\mathrm{a}}$ & 1.2 & $\mathrm{~kg} \cdot \mathrm{m}$ \\
$\sigma$ & 2.25 & - \\
\hline & &
\end{tabular}

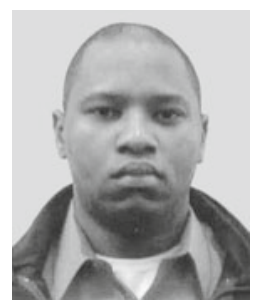

Erhun IYASERE was born in 1983, in Benin City, Nigeria. He received his B.S. degree in Electrical Engineering from the Citadel in 2004. In 2004, he enrolled at Clemson University, where he received his M.S. degree in December 2007, and Ph.D. degree in Electrical Engineering in May 2010. In July 2010, he joined the Jacobsen, a Textron Company as a design engineer. He is a member of the Institute of Electrical and Electronic Engineers and Tau
Beta Pi. Dr. Iyasere's primary research interests include nonlinear control techniques for mechatronic systems. E-mail: eiyasere@ @extron.com.

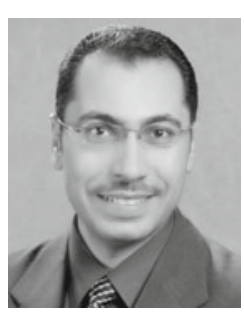

Mohammed H. SALAH received his B.S. degree in Electrical Engineering from Al-Balqaa Applied University, Amman, Jordan, M.S. degree in Electrical Engineering from University of New Hampshire, Durham, NH, U.S.A., and Ph.D. degree in Electrical Engineering from Clemson University, Clemson, SC, U.S.A., in 2000, 2003, and 2007, respectively. From 2000 to 2002, he was a control engineer at PALCO for automation and electronic control, Amman, Jordan. In August 2007, he joined the Department of Mechatronics Engineering at Hashemite University, Zarqa, Jordan, where he is currently an assistant professor. His current research interests include nonlinear control, mechatronics systems, control of MEMS, renewable energy systems, automotive systems, hydraulic and pneumatic control systems, and intelligent robotic systems. E-mail: msalah@hu.edu.jo.

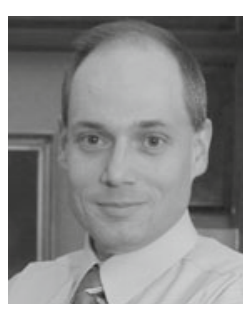

Darren M. DAWSON received his B.S. degree in Electrical Engineering from the Georgia Institute of Technology in 1984. He then worked for Westinghouse as a control engineer from 1985 to 1987. In 1987, he returned to the Georgia Institute of Technology, where he received his Ph.D. degree in Electrical Engineering in March 1990. In July 1990, he joined the Electrical and Computer Engineering (ECE) Department at Clemson University, where he currently holds the position of McQueen Quattlebaum Professor. From 2005 to 2007, he also served as the ECE Department Graduate Coordinator. As of August 2007, he has held the position of ECE Department Chair. His research interests include nonlinear control techniques for mechatronic systems such as electric machinery, robotic manipulator systems, overhead cranes, magnetic bearings, vision-based systems, mobile platforms (underwater vehicles, surface ships, satellites, aircraft, etc.), and mechanical friction. E-mail: ddarren@clemson.edu.

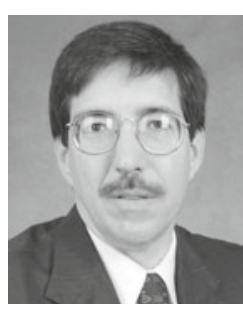

John R. WAGNER received his B.S., M.S., and $\mathrm{Ph} . \mathrm{D}$. degrees in Mechanical Engineering from the State University of New York at Buffalo and Purdue University. Dr. Wagner was on the technical staff at Delco Electronics and Delphi Automotive Systems prior to joining the Department of Mechanical Engineering at Clemson University. His research interests include nonlinear control theory, behavioral modeling, diagnostic and prognostic strategies, and mechatronic system design with application to automotive and wind turbine systems. Professor Wagner is a fellow of the American Society of Mechanical Engineers (ASME) and a register professional engineer. E-mail: jwagner@clemson.edu.

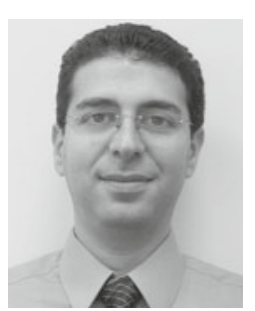

Enver TATLICIOGLU received his B.S. degree in Electrical and Electronics Engineering from Dokuz Eylul University, Izmir, Turkey, and the Ph.D. degree in Electrical and Computer Engineering from Clemson University, Clemson, SC, U.S.A. in 1999 and 2007, respectively. Upon completion of his $\mathrm{Ph} . \mathrm{D}$. degree, he worked as a postdoctoral research fellow at the Department of Electrical and Computer Engineering, Clemson University, then joined the Electrical \& Electronics Engineering Department at Izmir Institute of Technology, Izmir, Turkey where he is currently an assistant professor. His research interests include robust, adaptive, and optimal control of nonlinear systems, predictor-based control of uncertain nonlinear systems, output feedback control, nonlinear control techniques for kinematically redundant robot manipulators, haptic systems and teleoperation, and dynamic modelling of extensible continuum robot manipulators. E-mail: envertatlicioglu@iyte.edu.tr. 